\title{
Galactic archaeology with asteroseismology and spectroscopy: Red giants observed by CoRoT and APOGEE`
}

\author{
F. Anders ${ }^{1,2}$, C. Chiappini ${ }^{1,2}$, T. S. Rodrigues ${ }^{2,3,4}$, A. Miglio ${ }^{5}$, J. Montalbán ${ }^{4}$, B. Mosser ${ }^{6}$, L. Girardi ${ }^{2,3}$, M. Valentini ${ }^{1}$, \\ A. Noels ${ }^{7}$, T. Morel ${ }^{7}$, J. A. Johnson ${ }^{8}$, M. Schultheis ${ }^{9}$, F. Baudin ${ }^{10}$, R. de Assis Peralta ${ }^{6}$, S. Hekker ${ }^{11,12}$, \\ N. Themeß111,12, T. Kallinger ${ }^{13}$, R. A. García ${ }^{14}$, S. Mathur ${ }^{15}$, A. Baglin ${ }^{6}$, B. X. Santiago ${ }^{2,16}$, M. Martig ${ }^{17}$, I. Minchev ${ }^{1}$, \\ M. Steinmetz ${ }^{1}$, L. N. da Costa ${ }^{2,18}$, M. A. G. Maia ${ }^{2,18}$, C. Allende Prieto ${ }^{19,20}$, K. Cunha ${ }^{18}$, T. C. Beers ${ }^{21}$, C. Epstein ${ }^{8}$, \\ A. E. García Pérez ${ }^{19,20}$, D. A. García-Hernández ${ }^{19,20}$, P. Harding ${ }^{22}$, J. Holtzman $^{23}$, S. R. Majewski ${ }^{24}$, \\ Sz. Mészáros ${ }^{25,26}$, D. Nidever ${ }^{27}$, K. Pann $^{23,28}$, M. Pinsonneault ${ }^{8}$, R. P. Schiavon ${ }^{29}$, D. P. Schneider ${ }^{30,31}$, \\ M. D. Shetrone ${ }^{32}$, K. Stassun ${ }^{33}$, O. Zamora ${ }^{19,20}$, and G. Zasowski ${ }^{34}$
}

(Affiliations can be found after the references)

Received 17 August 2015 / Accepted 3 August 2016

\begin{abstract}
With the advent of the space missions CoRoT and Kepler, it has recently become feasible to determine precise asteroseismic masses and relative ages for large samples of red giant stars. We present the CoRoGEE dataset, obtained from CoRoT light curves for 606 red giants in two fields of the Galactic disc that have been co-observed by the Apache Point Observatory Galactic Evolution Experiment (APOGEE). We used the Bayesian parameter estimation code PARAM to calculate distances, extinctions, masses, and ages for these stars in a homogeneous analysis, resulting in relative statistical uncertainties of $\lesssim 2 \%$ in distance, $\sim 4 \%$ in radius, $\sim 9 \%$ in mass and $\sim 25 \%$ in age. We also assessed systematic age uncertainties stemming from different input physics and mass loss. We discuss the correlation between ages and chemical abundance patterns of field stars over a broad radial range of the Milky Way disc $\left(5 \mathrm{kpc}<R_{\mathrm{Gal}}<14 \mathrm{kpc}\right)$, focussing on the $[\alpha / \mathrm{Fe}]-[\mathrm{Fe} / \mathrm{H}]$-age plane in five radial bins of the Galactic disc. We find an overall agreement with the expectations of pure chemical-evolution models computed before the present data were available, especially for the outer regions. However, our data also indicate that a significant fraction of stars now observed near and beyond the solar neighbourhood migrated from inner regions. Mock CoRoGEE observations of a chemodynamical Milky Way disc model indicate that the number of high-metallicity stars in the outer disc is too high to be accounted for even by the strong radial mixing present in the model. The mock observations also show that the age distribution of the $[\alpha /$ Fe $]$-enhanced sequence in the CoRoGEE inner-disc field is much broader than expected from a combination of radial mixing and observational errors. We suggest that a thick-disc/bulge component that formed stars for more than $3 \mathrm{Gyr}$ may account for these discrepancies. Our results are subject to future improvements due to (a) the still low statistics, because our sample had to be sliced into bins of Galactocentric distances and ages; (b) large uncertainties in proper motions (and therefore guiding radii); and (c) corrections to the asteroseismic mass-scaling relation. The situation will improve not only upon the upcoming Gaia data releases, but also with the foreseen increase in the number of stars with both seismic and spectroscopic information.
\end{abstract}

Key words. asteroseismology - stars: fundamental parameters - Galaxy: abundances - Galaxy: disk - Galaxy: evolution

\section{Introduction}

To reconstruct the formation history of the Milky Way, one would ideally like to obtain precise and unbiased ages for thousands or millions of stars in all parts of our Galaxy. To date, this goal is still far beyond reach, at least until astrometric parallaxes from the Gaia satellite (Perryman et al. 2001) and asteroseismic data from K2 (Howell et al. 2014) and PLATO 2.0 (Rauer et al. 2014) will become available.

A common work-around for this problem is to use relative "chemical clocks" provided by element abundance ratios (Pagel 1997; Matteucci 2001): each star carries in its atmosphere the enrichment history of the gas from which it was formed, only minimally polluted by its own stellar evolution, and accessible through spectroscopy. By combining this wealth of information with kinematic properties of stellar populations in different Galactic environments, we can systematically unravel the importance of the various physical processes that led to the

\footnotetext{
$\star$ The data described in Table B.1 are only available at the CDS via anonymous ftp to cdsarc.u-strasbg. fr (130.79.128.5) or via http://cdsarc.u-strasbg.fr/viz-bin/qcat?J/A+A/597/A30
}

formation of the Milky Way as we see it today ("Galactic archaeology"; Freeman \& Bland-Hawthorn 2002; Turon et al. 2008).

Still, age determinations provide crucial constraints on several astrophysical processes: For example, the ages of old halo stars can be used as a lower limit for the age of the Universe (Hill et al. 2002). The Galactic age-metallicity relation (e.g. Twarog 1980; Edvardsson et al. 1993; Ng \& Bertelli 1998), the star-formation history (Gilmore 1999) or the evolution of abundance gradients (e.g. Carraro et al. 1998; Chen et al. 2003) are essential tools for understanding the evolution of our Milky Way.

During the past ten years, ever more sophisticated chemodynamical models of Milky-Way-mass galaxies have been developed in a cosmological context (e.g. Abadi et al. 2003; Stinson et al. 2010; Guedes et al. 2011; Brook et al. 2012; Scannapieco et al. 2015; Roca-Fàbrega et al. 2016). However, detailed models that match many of the Milky Way's chemo-dynamical correlations (Minchev et al. 2013, 2014b) are still rare (see discussions in Scannapieco et al. 2012 and Minchev et al. 2013). These can be compared to observations, but it is often difficult to find observables that are powerful 
enough to discard certain scenarios of the Galaxy's evolution. With the availability of age estimates for large stellar samples - even if they are only valid in a relative sense - this situation changes drastically.

It is therefore important to revisit the full age-chemistrykinematics space with samples that cover larger portions of the Galactic disc. In this high-dimensional space, we can then look for robust statistical relations that realistic models have to fulfil. With the joint venture of asteroseismology and spectroscopic surveys, we are now in a position to constrain key parameters of stellar and Galactic evolution.

Unlike stellar radii and masses, the ages of stars cannot be directly measured, only inferred through modelling. Among the various available stellar age indicators (e.g. Li abundance, U/Th ratio, stellar activity, rotation, X-ray luminosity, and position in the Hertzsprung-Russell diagram), one of the most promising methods that can deliver reliable age estimates for a wide range of ages is the comparison of measured atmospheric and asteroseismic parameters of evolved stars with models of stellar evolution (e.g. Miglio 2012).

It is well-known (e.g. Ulrich 1986; Christensen-Dalsgaard 1988) that detailed asteroseismic analyses involving individual oscillation frequencies may deliver precise age determinations. Depending on the spectral type of the star, a number of seismic characteristics can be used to investigate the stellar interior and infer an age estimate. However, this so-called "boutique" or "à la carte modelling" (Soderblom 2013; Lebreton et al. 2014) requires extremely accurate measurements of several pulsation modes. To date, this is only possible for the Sun (e.g. Gough 2001) and a relatively small number of bright dwarf stars observed by CoRoT and Kepler (e.g. Metcalfe et al. 2010; Batalha et al. 2011; Mathur et al. 2012; Silva Aguirre et al. 2013; Chaplin \& Miglio 2013; Lebreton et al. 2014; Metcalfe et al. 2014).

For large samples of red giant stars (first-ascent red giants as well as red-clump stars), statistical studies follow a different approach called "ensemble asteroseismology" (e.g. Chaplin et al. 2011). This method typically focusses on two main seismic characteristics of the frequency spectrum of solarlike oscillating giants: the large frequency separation $\Delta v$, related to the stellar mean density (Tassoul 1980; Ulrich 1986; Christensen-Dalsgaard 1993), and the frequency of maximum oscillation power $v_{\max }$, related to the acoustic cut-off frequency (Brown et al. 1991; Kjeldsen \& Bedding 1995; Belkacem et al. 2011). The mass and radius of a star have been shown to scale with these quantities via:

$$
\begin{aligned}
\frac{M}{M_{\odot}} & \simeq\left(\frac{v_{\text {max }}}{v_{\max , \odot}}\right)^{3}\left(\frac{\Delta v}{\Delta v_{\odot}}\right)^{-4}\left(\frac{T_{\mathrm{eff}}}{T_{\mathrm{eff}, \odot}}\right)^{3 / 2}, \\
\frac{R}{R_{\odot}} & \simeq\left(\frac{v_{\max }}{v_{\max , \odot}}\right)\left(\frac{\Delta v}{\Delta v_{\odot}}\right)^{-2}\left(\frac{T_{\mathrm{eff}}}{T_{\mathrm{eff}, \odot}}\right)^{1 / 2},
\end{aligned}
$$

where $T_{\text {eff }}$ is the star's effective temperature, and the solar values $\Delta v_{\odot}=135.03 \mu \mathrm{Hz}, v_{\max , \odot}=3140.0 \mu \mathrm{Hz}$, and $T_{\text {eff } \odot}=5780 \mathrm{~K}$ (Pinsonneault et al. 2014) are used in the following.

The scaling relations (1) have been tested with independent methods in the past years (eclipsing binaries, open clusters, interferometry, HIPPARcos parallaxes), and shown to be valid for a broad parameter regime (see Chaplin \& Miglio 2013 for a review). Possible systematic biases concerning the mass determination are introduced by departures from a simple scaling of $\Delta v$ with the square root of the stellar mean density (see e.g. White et al. 2011; Miglio 2012; Miglio et al. 2013b; Belkacem et al. 2013). Suggested corrections to the $\Delta v$ scaling probably depend (to a level of a few percent) on the stellar structure itself. Moreover, the average $\Delta v$ is known to be affected (to a level of around $1 \%$ in the Sun) by inaccurate modelling of nearsurface layers.

The seismic mass of a red giant provides a powerful constraint on its age, because its red-giant branch (RGB) lifetime is relatively short compared to its main-sequence lifetime. Combined with independent measurements of metallicity and effective temperature, the main seismic characteristics provide good statistical measures for the primary derived parameters of a star, such as mass, radius, distance, and age (e.g. Miglio 2012; Rodrigues et al. 2014; Casagrande et al. 2016).

Unfortunately, the overall quality (in terms of precision and accuracy) of age determinations for giant stars is still fairly limited (e.g. Jørgensen \& Lindegren 2005; Soderblom 2010; Casagrande et al. 2016). Systematic age uncertainties depend on the quality of the observables and on theoretical uncertainties of stellar models (e.g. Noels \& Bragaglia 2015; see also Sect. 3.2.2)

With the recently established synergy of asteroseismology and high-resolution spectroscopy surveys, it has become possible to determine more precise ages for red giants.

The detection of solar-like oscillations in thousands of field stars by CoRoT and Kepler has opened the door to detailed studies of the Milky Way's stellar populations. Data from the first CoRoT observing run revealed solar-like oscillations in thousands of red giants (Hekker et al. 2009). Miglio et al. (2009) presented a first comparison between observed and predicted seismic properties of giants in the first CoRoT field, which highlighted the expected signatures of red-clump stars in the $\Delta v$ and $v_{\max }$ distributions. Miglio et al. (2013b) presented a first comparison between populations of red giants observed by CoRoT in two different parts of the Milky Way (the CoRoT fields LRa01 and LRc01 also investigated here; see Fig. 1), which showed significant differences in the mass distributions of these two samples, and were interpreted as mainly due to the vertical gradient in the distribution of stellar masses (hence ages) in the disc (see also Casagrande et al. 2016 for a first measurement of the vertical disc age gradient). However, the precision of the age determinations used in this pilot study was still limited to $30-40 \%$, due to the absence of constraints on photospheric chemical composition (Miglio et al. 2013a).

Recently, large-scale follow-up observations of seismic targets have begun. The SAGA project (Casagrande et al. 2014, 2016) is covering the Kepler field with Strömgren photometry, thereby obtaining more precise stellar parameters. Similarly, spectroscopic stellar surveys such as RAVE (Steinmetz et al. 2006), APOGEE (Majewski et al. 2016), the Gaia-ESO survey (Gilmore 2012), LAMOST (Zhao et al. 2012), and GALAH (Zucker et al. 2012) are observing CoRoT and Kepler targets to anchor their spectroscopic surface gravity and distance measurements (e.g. Bovy et al. 2014; Holtzman et al. 2015) - and to ultimately use the combined datasets to constrain the chemodynamical evolution of the Milky Way. The CoRoT-APOGEE (CoRoGEE) dataset paves the way for future advances in this direction.

Our paper is structured as follows: the CoRoGEE sample and the provenance of the different data (asteroseismology, spectroscopy, photometry and astrometry) are presented in Sect. 2. Section 3 summarises our analysis and leads to our estimates of the main stellar "desirables", such as mass, radius, age, distance, extinction, and kinematical parameters. We emphasise that our 


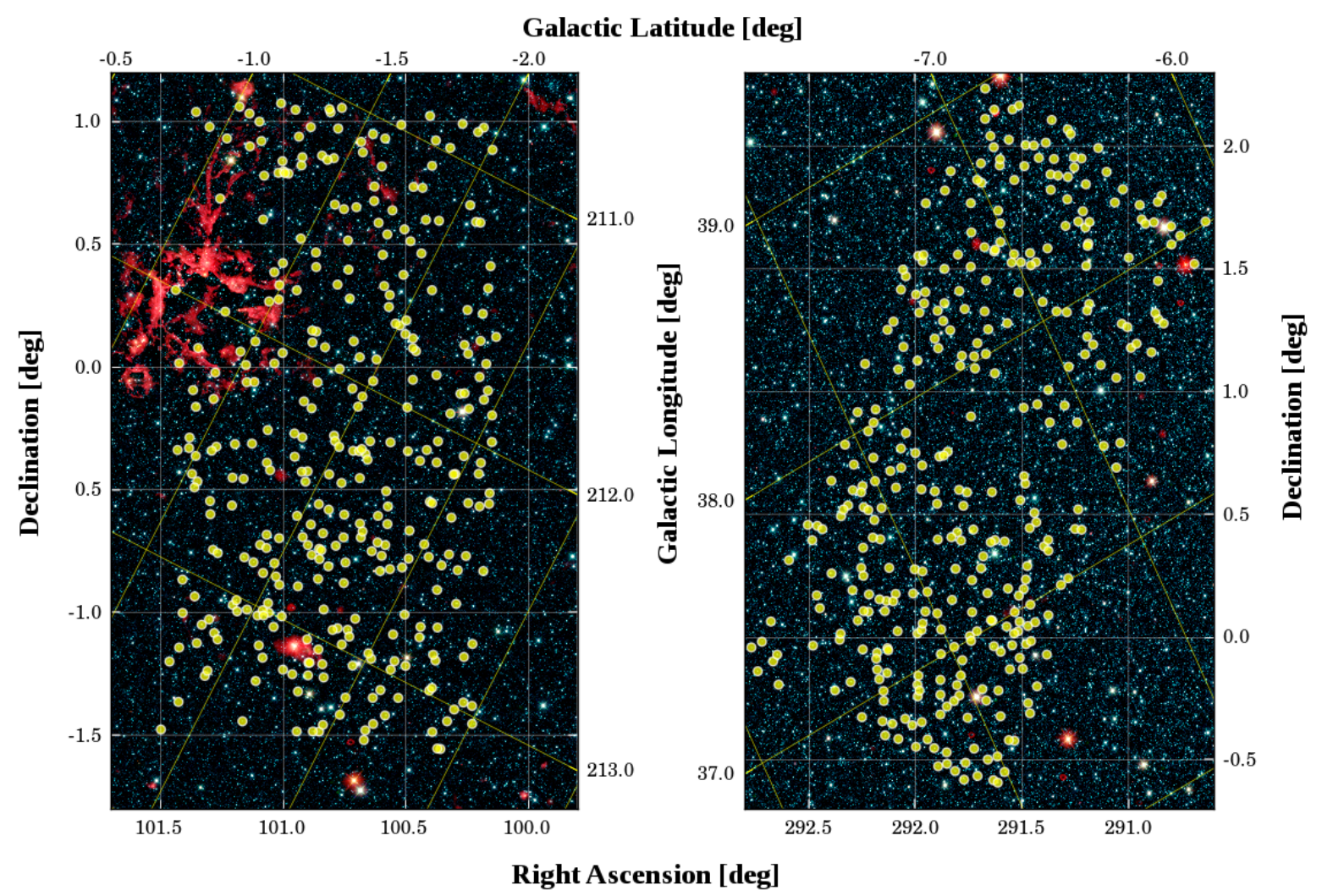

Fig. 1. Location of the stars observed with APOGEE in the two CoRoT exoplanet fields LRa01 (left) and LRc01 (right). Indicated in yellow are the stars for which asteroseismic parameters were available. The background colour image is composed of near-infrared WISE $W 1, W 2$ and W3 images from the AllWISE data release (Cutri et al. 2013). Bottom and outside vertical labels display equatorial coordinates, while the top and central vertical labels refer to Galactic coordinates. Corresponding coordinate grids are also shown.

age estimates should be considered relative age indicators that are to be used in a statistical sense only.

In Sect. 4, we use our sample to study for the first time the variation of the $[\alpha / \mathrm{Fe}]-\mathrm{vs} .-[\mathrm{Fe} / \mathrm{H}]^{1}$ relation with Galactocentric distance in three broad age bins, and compare our data with predictions from a chemodynamical Galaxy model. We conclude and discuss future paths to improve our analysis in Sect. 5.

The CoRoGEE dataset covers a wide radial range of the Galactic disc and provides precise stellar parameters, distances, and chemical abundances. Therefore, the presented data provide material for a number of subsequent analyses. In two companion papers, we focus on specific results: 1. the discovery of an apparently young stellar population with enhanced $[\alpha / \mathrm{Fe}]$ ratio (Chiappini et al. 2015), and 2. the variation of the disc radial metallicity profile with stellar age (Anders et al. 2016a). The data are publicly available at the CDS (see Appendix B).

\section{Observations}

Our observations combine the global asteroseismic parameters derived from precision light curves obtained by the CoRoT satellite (Baglin et al. 2006; Michel et al. 2008) with stellar parameters and chemical abundances inferred from near-infrared (NIR) high-resolution spectra taken by the Apache Point Observatory Galactic Evolution Experiment (APOGEE). The field maps of

1 The abundance ratio of two chemical elements $X$ and $Y$ is defined as $[X / Y]=\lg \frac{n_{X}}{n_{Y}}-\lg \left(\frac{n_{X}}{n_{Y}}\right)_{\odot}$, where $n_{X}$ and $n_{Y}$ are respectively the numbers of nuclei of elements $X$ and $Y$, per unit volume in the stellar photosphere. the two CoRoT fields observed with APOGEE are shown in Fig. 1. An overview on the data assembly and analysis is given in Fig. 2.

\subsection{Adopted seismic parameters}

The CoRoT data used in this work are a subset of the data analysed by Mosser et al. (2010) and Miglio et al. (2013b): The CoRoT long runs in the LRa01 and LRc01 exoplanet fields comprise photometric time series for several thousand stars of about 140 days, resulting in a frequency resolution of $\sim 0.08 \mu \mathrm{Hz}$. For stars with detectable solar-like oscillations, Mosser et al. (2010) determined the large frequency separation, $\Delta v$, and the frequency of maximum oscillation power, $v_{\max }$, from the frequency spectra with the envelope autocorrelation-function method (Mosser \& Appourchaux 2009), but without reporting individual uncertainties for these quantities.

In the following, we use the seismic parameters obtained from CoRoT N2 light curves ${ }^{2}$ in the same way as in Mosser et al. (2010), updated to deliver individual uncertainties on $\Delta v$ and $v_{\max }$. When the envelope autocorrelation signal is high enough, a more precise estimate of the large separation is provided by the use of the so-called universal pattern method (Mosser et al. 2011). A comprehensive data release of newly reduced CoRoT light curves and higher-level science products, using analyses of several different seismic pipelines, will be presented in a separate paper.

\footnotetext{
2 http://idoc-corot.ias.u-psud.fr/jsp/doc/ DescriptionN2v1.3.pdf
} 


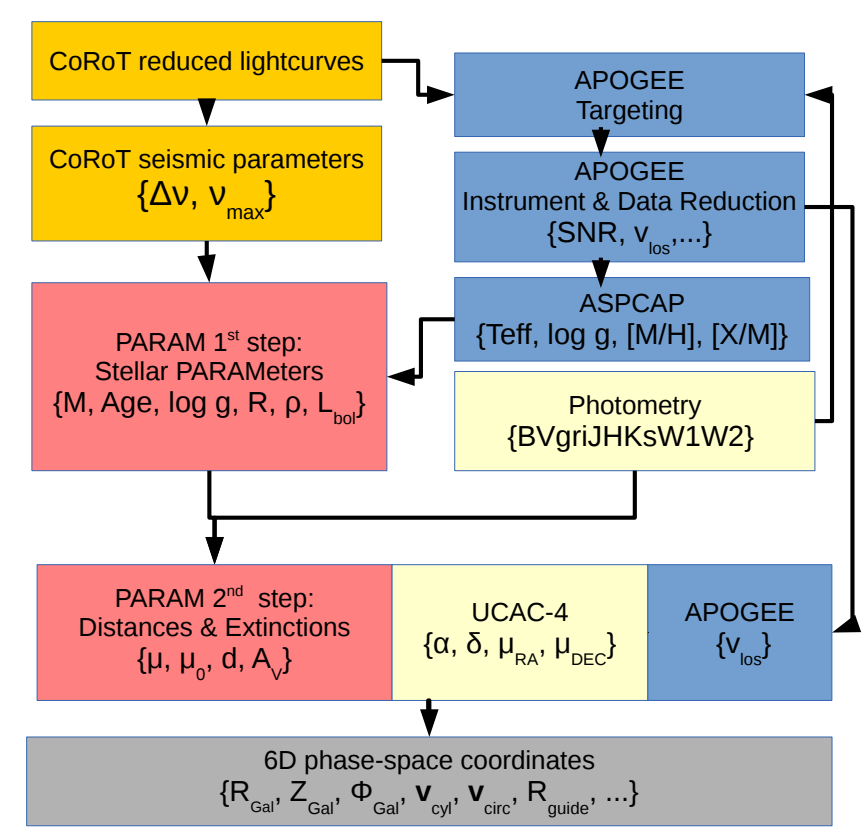

Fig. 2. Overview of the data provenance and analysis steps performed for the CoRoT-APOGEE (CoRoGEE) data. Blue boxes correspond to APOGEE data products, orange boxes to CoRoT data, and light yellow boxes to existing catalogue data. Red boxes summarise the two parts of the PARAM pipeline, while the grey box summarises the kinematical data used for this work.

As shown in Mosser et al. (2010) and Miglio et al. (2013b), the target selection for the CoRoT asteroseismology program is homogeneous in both fields: solar-like oscillations were searched for in giant stars obeying the following cuts in the colour-magnitude diagram: $K_{\mathrm{s}}<12,0.6<J-K_{\mathrm{s}}<1.0$. Mosser et al. (2010) also demonstrated that, for a wide parameter range, the selection bias introduced by the additional requirement of detected oscillations does not measurably affect the $\Delta v$ or $v_{\max }$ distributions in the two fields.

\subsection{Spectroscopic data}

APOGEE (Majewski et al. 2016) is a Galactic archaeology experiment operating during the third and fourth epochs of the Sloan Digital Sky Survey (SDSS-III, Eisenstein et al. 2011; SDSS-IV). It uses the $2.5 \mathrm{~m}$ telescope at APO (Gunn et al. 2006) to feed a multi-object NIR fiber spectrograph (Wilson et al. 2010, 2012) that delivers high-resolution $(R \sim 22500) H$-band spectra $(\lambda=1.51-1.69 \mu \mathrm{m})$ of mostly red giants. Dedicated processing and analysis pipelines (Nidever et al. 2015; Holtzman et al. 2015) allow for the determination of precise $(\sim 100 \mathrm{~m} / \mathrm{s})$ and accurate $(\sim 350 \mathrm{~m} / \mathrm{s})$ radial velocities. In addition, the APOGEE Stellar Parameter and Chemical Abundances Pipeline (ASPCAP; García Pérez et al. 2015) provides stellar parameters and elemental abundances of 15 chemical elements from the best fit over extensive grids of pre-calculated synthetic stellar spectra (Zamora et al. 2015) to the observed spectra.

As an SDSS-III/APOGEE ancillary program, 690 stars with detected seismic oscillations in the two CoRoT exoplanet fields LRa01 (APOGEE fields COROTA and COROTA3; $(l, b)_{\mathrm{cen}}=$ $(212,-2))$ and LRc01 (COROTC; $\left.(l, b)_{\text {cen }}=(37,-7)\right)$ were observed with the APOGEE instrument, at high signal-to-noise ratios (median $S / N$ of 230 per resolution element). The field maps of the observed targets are shown in Fig. 1. The APOGEE targeting scheme allows for the combination of spectra taken at different times, so-called visits. Most of the stars $(\sim 80 \%)$ have been observed at least three times to reach the signal-to-noise ratio goal of 100 , which is necessary to infer precise chemical abundance information (Zasowski et al. 2013).

Unfortunately, the actual target selection for APOGEE observations of CoRoT solar-like oscillating red giants has not been carried out on the basis of a simple selection function. The targets on the plates observed by APOGEE are a mixture of:

1. solar-like oscillating stars identified by Mosser et al. (2011) - preferentially selected to be RGB stars;

2. CoRoT stars observed by the Gaia-ESO survey (Gilmore 2012) for the purpose of cross-calibration; and

3. APOGEE main-survey targets that were found to show solarlike oscillations in CoRoT, but were not selected on that basis.

Therefore, the best way to correct for the CoRoGEE selection function is to compare what was observed with what could have been observed (i.e., compare the resulting spectro-seismic sample with the underlying photometric sample). In addition, it is necessary to assess whether the photometric parent sample (red giants in the fields LRa01 and LRc01) is representative of the overall stellar content in these fields (as done in Miglio et al. 2013 ba). Both steps can be accomplished with stellar population synthesis modelling (see Anders et al. 2016b). One intermediate selection effect that we cannot address with the current CoRoGEE sample is whether the red giants with detected solar-like oscillations are fully representative of the underlying population. For the Kepler field, Casagrande et al. (2016) found that this is only true for a narrower region in the colour-magnitude diagram than we are considering here; our giant sample may therefore be slightly biased against redder colours (more evolved stars).

For this work, we make use of the ASPCAP-derived stellar parameters effective temperature, $T_{\text {eff }}$, scaled-solar metallicity, $[\mathrm{M} / \mathrm{H}]$, and relative $\alpha$-element abundance, $[\alpha / \mathrm{M}]$, from the SDSS data release 12 (DR12 Alam et al. 2015; Holtzman et al. $2015)^{3}$. For the comparison to stellar isochrones, we approximated the overall metal abundance by the sum $[\mathrm{Z} / \mathrm{H}] \simeq$ $[\mathrm{M} / \mathrm{H}]_{\text {uncalib }}+[\alpha / \mathrm{M}]_{\text {uncalib }}$ (e.g. Salaris et al. 1993; Anders et al. 2014). Figure 3 summarises the distribution of the CoRoGEE stars in ASPCAP parameter space. We used calibrated values for the ASPCAP $T_{\text {eff }}$ and surface gravity $\log g$.

To ensure that the ASPCAP stellar parameters and chemical abundances do not suffer from unknown problems, we discarded 12 stars that did not satisfy the high-quality criteria laid out in Anders et al. (2014). We also flagged and removed 14 stars for which a visual inspection of the CoRoT light curves revealed spurious detection of solar-like oscillations. In addition, we required that the difference between the spectroscopically derived surface gravity be not too far from the value predicted by the seismic scaling relations: $\left|\log g_{\mathrm{ASPCAP}}^{\text {calib }}-\log g_{\text {seismo }}\right|<0.5$ dex. This criterion removed 47 stars for which the ASPCAP solution is incompatible with the seismic measurements (crosses in Fig. 4, left panel). In addition, 11 stars were rejected by our stellar parameter pipeline because their measured input values $\left\{\Delta v, v_{\max }, T_{\text {eff }},[\mathrm{M} / \mathrm{H}]\right\}$ were incompatible with any stellar model within their uncertainties (crossed circles in Fig. 4, right panel).

\footnotetext{
3 We estimate the uncertainties in these abundances as $\sigma[\mathrm{M} / \mathrm{H}]=$ $\sigma[\mathrm{Fe} / \mathrm{H}]$ and $\sigma[\alpha / \mathrm{M}]=\sqrt{\sigma[\mathrm{Mg} / \mathrm{H}]^{2}+\sigma[\mathrm{Fe} / \mathrm{H}]^{2}}$.
} 

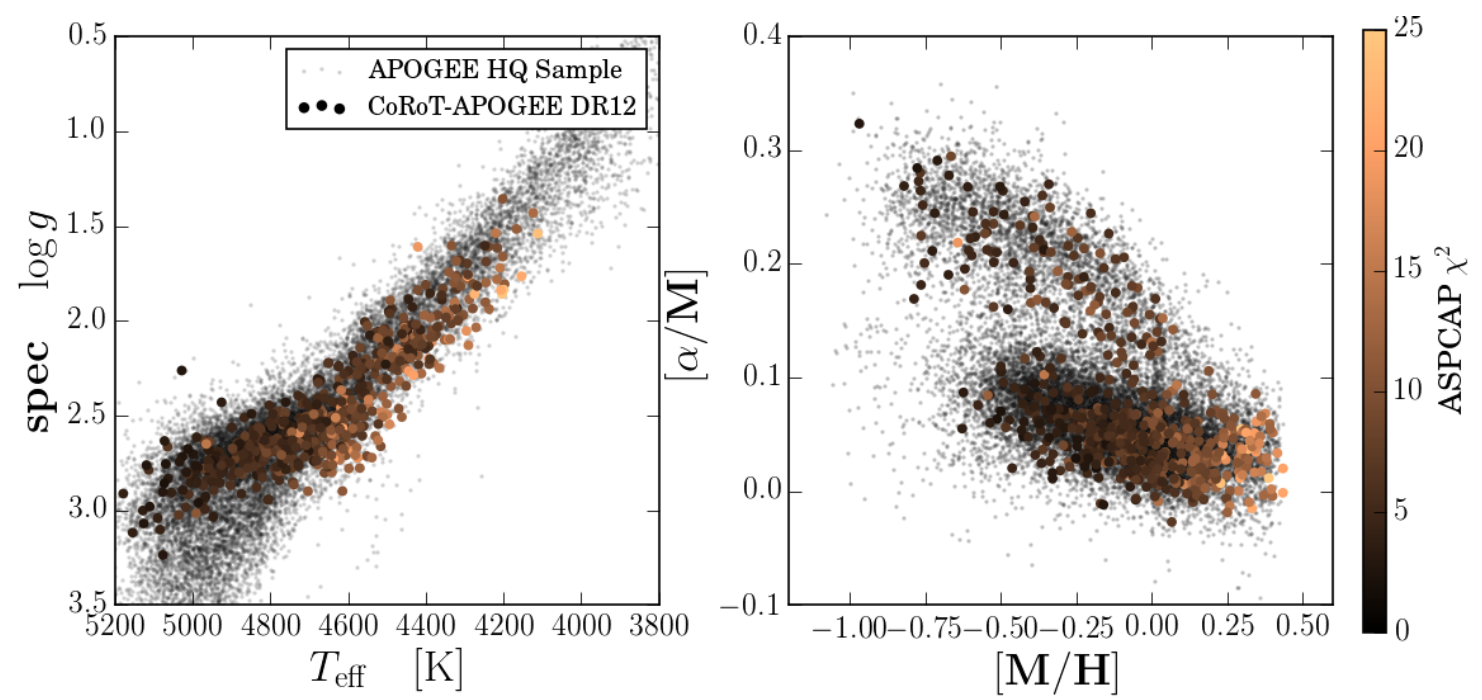

Fig. 3. Location of the CoRoT-APOGEE stars in the ASPCAP $\log g$-vs.- $T_{\text {eff }}$ Kiel diagram $($ left $)$ and the $[\alpha / \mathrm{Fe}]-\mathrm{vs}$.-[Fe/H] chemical abundance plane. The colour encodes the reduced $\chi^{2}$ of the ASPCAP fit. In the background, we plot the APOGEE DR10 high-quality giant sample (Anders et al. 2014), updated to DR12 atmospheric parameters, for comparison.


Fig. 4. Left: difference between ASPCAP (calibrated) $\log g$ and gravity determined from seismic scaling relations, as a function of effective temperature. Stars located in the grey-shaded area $\left(\left|\log g_{\mathrm{ASPCAP}}-\log g_{\text {seismo }}\right|>0.5 \mathrm{dex}\right.$; blue crosses) were excluded from the analysis. Right: $v_{\max }-\Delta v$ diagram for our program stars. In addition to the $\log g$ consistency requirement, nine stars (mostly located far from the $v_{\max }-\Delta v$ sequence; blue crossed circles) were also rejected by the PARAM pipeline. Error bars in the upper part of the diagrams represent average uncertainties.

\subsection{Photometry and astrometry}

To determine distances to the stars in our sample with the best possible precision, the spectroscopic and asteroseismic information was complemented by photometric data obtained over a wide wavelength range.

Standard Harris $B$ and $V$ as well as Sloan-Gunn $r^{\prime}$ and $i^{\prime}$ magnitudes are available for our CoRoT targets from the OBSCAT catalogue which was released as a supplement to the EXODAT archive (Meunier et al. 2007; Damiani et al. 2016). The observations were performed with the Wide Field Camera (WFC) at the $2.5 \mathrm{~m}$ Isaac Newton Telescope (INT) at Roque de los Muchachos Observatory (La Palma) in $2002^{4}$.

Because the photometry of the USNO-B catalogue (which is also provided by EXODAT) is based on digitised photographic

\footnotetext{
4 http://cesam.oamp.fr/exodat/index/ exodat-documentation\#Photometryavailableforsubsamples
}

Schmidt plates and its calibration suffers from inaccuracies and inhomogeneities of about 0.2 mag (Monet et al. 2003), we refrained from using this database.

We also added Johnson $B V$ and Sloan $g^{\prime} r^{\prime} i^{\prime}$ photometry from the APASS survey's 6th data release (Henden \& Munari 2014), with photometric accuracies of about $0.02 \mathrm{mag}$.

In the infrared, accurate $J H K_{\mathrm{s}}$ photometry is available from the 2MASS Point Source Catalog (Cutri et al. 2003), which served as the major input catalogue for APOGEE. We also added WISE $W 1 W 2$ filters from the AllWISE Catalog (Cutri et al. 2013) for which the photometric precision is sufficient to constrain the mid-infrared region of the stellar spectral energy distribution ${ }^{5}$.

\footnotetext{
5 As in Rodrigues et al. (2014), we discard the filters W3 and W4 because of possible contamination by warm interstellar dust (e.g. Davenport et al. 2014) and larger measurement uncertainties.
} 

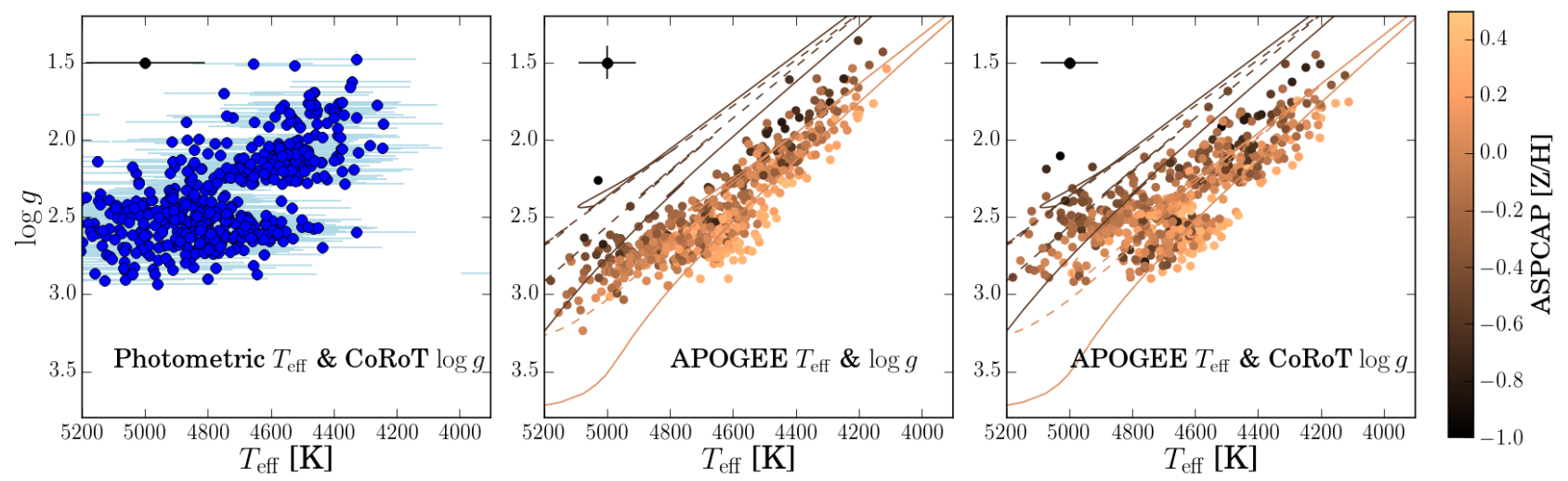

Fig. 5. $\log g-T_{\text {eff }}$ Kiel diagrams. Left: photometric temperatures and $\log g$ from CoRoT seismic parameters + scaling relations. Middle: purely spectroscopic diagram using APOGEE stellar parameters, colour-coded by metallicity. Right: joint CoRoT-APOGEE Kiel diagram. Overplotted are PARSEC isochrones (Bressan et al. 2012) for metallicities -0.6 and 0.0 at ages 1.4 (dashed lines) and 4.5 Gyr (solid lines) for comparison. As noted by Martig et al. (2015), there is a slight temperature discrepancy between models and data for sub-solar metallicities. Error bars in the upper left of each panel indicate median uncertainties.

Table 1. Summary of the number of CoRoT-APOGEE stars satisfying different quality criteria.

\begin{tabular}{lc}
\hline \hline Sample criterion & Stars \\
\hline CoRoT-APOGEE stars & 690 \\
with good ASPCAP results & 678 \\
and good seismic results & 664 \\
and $\left|\log g_{\text {ASPCAP }}^{\text {calib }}-\log g_{\text {seismo }}\right|<0.5$ dex & 617 \\
\hline Converged stellar PARAMeters and distances & 606 \\
LRa01 & 281 \\
LRc01 & 325 \\
and reliable UCAC-4 proper motions $(\mathrm{OK}$ flag) & 504 \\
and good orbits $\left(\sigma\left(v_{\mathrm{T}}\right)<50 \mathrm{~km} \mathrm{~s}^{-1}\right)$ & 234 \\
\hline
\end{tabular}

For kinematical studies, proper motions were compiled from the recent UCAC-4 catalogue (Zacharias et al. 2013), using only astrometric data that meet several high-quality criteria encoded in the UCAC-4 flags ( $80 \%$ of the stars $)$, in the same manner as in Anders et al. (2014).

\section{Analysis}

\subsection{Masses, radii, and ages}

To derive primary stellar parameters such as mass, luminosity, radius, and age, we used the Bayesian parameter estimation code PARAM ${ }^{6}$ (da Silva et al. 2006) with the recent improvements presented in Rodrigues et al. (2014). The code uses standard grid-based modelling (see Chaplin \& Miglio 2013 and references therein for an overview) to estimate stellar properties by comparison with theoretical stellar models, in our case the PARSEC isochrone models (Bressan et al. 2012).

When computing the desired stellar parameters, PARAM naturally accounts for the statistical uncertainties in the input parameters $\left\{\Delta v, v_{\max }, T_{\mathrm{eff}},[\mathrm{Z} / \mathrm{H}]\right\}$, and transforms them into the posterior probability distribution in stellar model space. We therefore denote uncertainties that are reflected in the shape of stellar parameter probability distribution functions (PDFs)

\footnotetext{
6 http://stev.oapd.inaf.it/cgi-bin/param
}
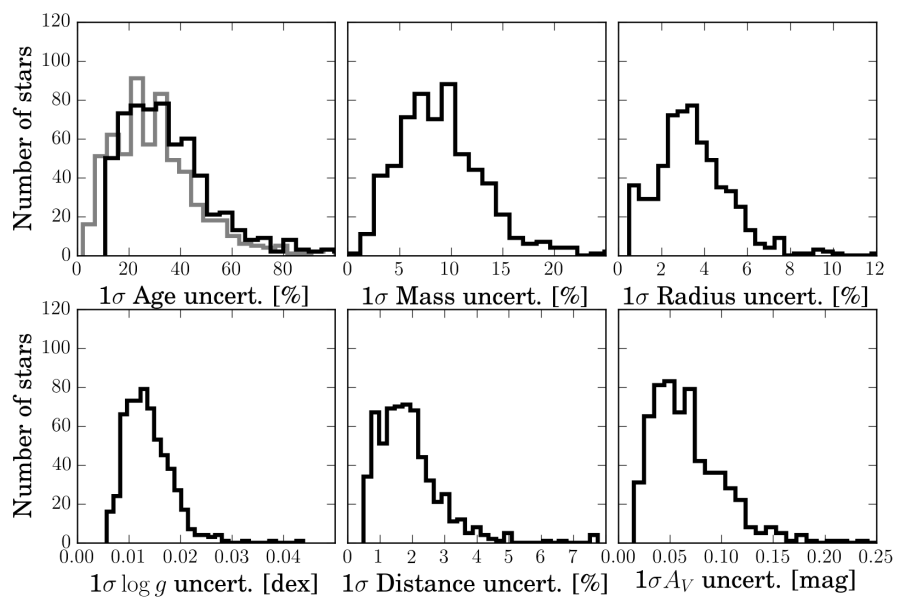

Fig. 6. Distributions of the $1 \sigma$-uncertainties in stellar age, mass, radius, $\log g$, distance, and extinction for our sample. For the ages (top left panel), we show the distributions of statistical (grey histogram) and total uncertainties.

statistical, because they arise from a (non-linear) propagation of uncertainties in the measured quantities ${ }^{7}$.

Stellar evolution models predict a rather tight relation between mass, metallicity, and age for red giants, with the age spread increasing with decreasing mass. Therefore, an uncertainty in stellar mass of about $10 \%$ typically results in a (statistical) age uncertainty of about 30\% (see e.g. Miglio et al. 2013b). In addition, depending on its location in the Hertzsprung-Russell diagram, a star may have broad or multi-peaked stellar parameter PDFs; the age PDFs show a wide variety of shapes. But even in the case of very broad PDFs, their shape does add valuable information: in the sense that we can quantify the knowledge we lack.

We therefore report the mode and $68 \%$ or $95 \%$ credible intervals of the marginalised PDF in mass, radius, age, distance, and extinction ${ }^{8}$ in our catalogue. We achieve typical statistical

\footnotetext{
7 Because we chose a particular set of isochrones, our statistical uncertainties are of course not model-independent.

8 Differently from Rodrigues et al. (2014), we computed these statistics from the interpolated PDF in linear units, and our formal $1 \sigma(2 \sigma)$ parameter uncertainties are defined as the smallest parameter interval around the mode that contain $68 \%$ (95\%) of the PDF.
} 
uncertainties of 0.015 dex in $\log g, 4 \%$ in radius, $9 \%$ in mass ${ }^{9}$, $25 \%$ in age, and $2 \%$ in distance (median values; see Fig. 6).

As discussed in the Introduction, stellar ages are by far more uncertain than any other classical stellar parameter and should be used only in a statistical, relative sense. This is due to a combination of the simple propagation of the stellar mass uncertainties with systematic uncertainties (mostly related to mass loss and the mass scaling relation). The magnitude of these uncertainties and their influence on stellar age estimates are discussed in Sect. 3.2.2. For a more detailed discussion of the systematic uncertainties involved in stellar modelling see Noels \& Bragaglia (2015), for instance.

\subsection{Age uncertainties: a closer look}

\subsubsection{Statistical uncertainties}

The age PDFs (which were not shown by Rodrigues et al. 2014) merit closer examination. Figure 7 shows the collection of all 606 age CoRoGEE PDFs, grouped in bins of mode age. Among them we find "well-behaved" (single-peaked) as well as more complex (double-, multi-peaked, very broad or grid-edgeaffected) stellar parameter PDFs.

It has been known for some time that isochrone-grid derived stellar age PDFs may show a great diversity (e.g. Takeda et al. 2007). As there is no straightforward way to classify or even quantify the behaviour of such diverse PDF shapes, the following numbers should be used with caution:

- Of the 606 stars passing all quality criteria, 246 display wellbehaved single-peaked age PDFs, 205 age PDFs are doublepeaked, 143 have three or more peaks, and 12 do not have local extrema because the PDF increases monotonically towards the upper age limit.

- Many of the multi-peaked PDFs have negligible PDF contributions from the secondary, tertiary etc. maxima, but a sizeable fraction exhibits genuinely complex function profiles.

- Figure 8 shows the distribution of stars classified according to the overall form of their age PDF in the HertzsprungRussell diagram. Multi-peaked age PDFs occur predominantly for stars with $\log g \simeq 2.4$, that is, parameter regions that are occupied by first-ascent RGB stars as well as redclump stars and asymptotic giant-branch (AGB) stars. The metallicity measurement does not add sufficient information to disentangle the different evolutionary stages. As noted by Rodrigues et al. (2014), the limiting factor is the accuracy of the effective temperatures, both in terms of models and measurements.

- An independent possibility of distinguishing between evolutionary phases (and thereby reducing the number of multi-peaked solutions) is offered by asteroseismology: Mosser et al. (2011) have measured mixed-mode period spacings (see also Bedding et al. 2011) for a fraction of the CoRoGEE targets (139 stars in LRc01, 28 stars in LRa01). This information was used to better constrain the age PDFs, as done in Rodrigues et al. 2014 for the APOGEEKepler (APOKASC) sample (Pinsonneault et al. 2014), and in Casagrande et al. $(2014,2016)$ for the SAGA survey.

\footnotetext{
9 Even in the very local volume the comparison of absolute magnitude (based on HIPPARCos parallax), $B-V$ colour, and [Fe/H] with stellar evolution models yields typical uncertainties in radius and mass of $6 \%$ and 8\%, respectively (Allende Prieto \& Lambert 1999).
}

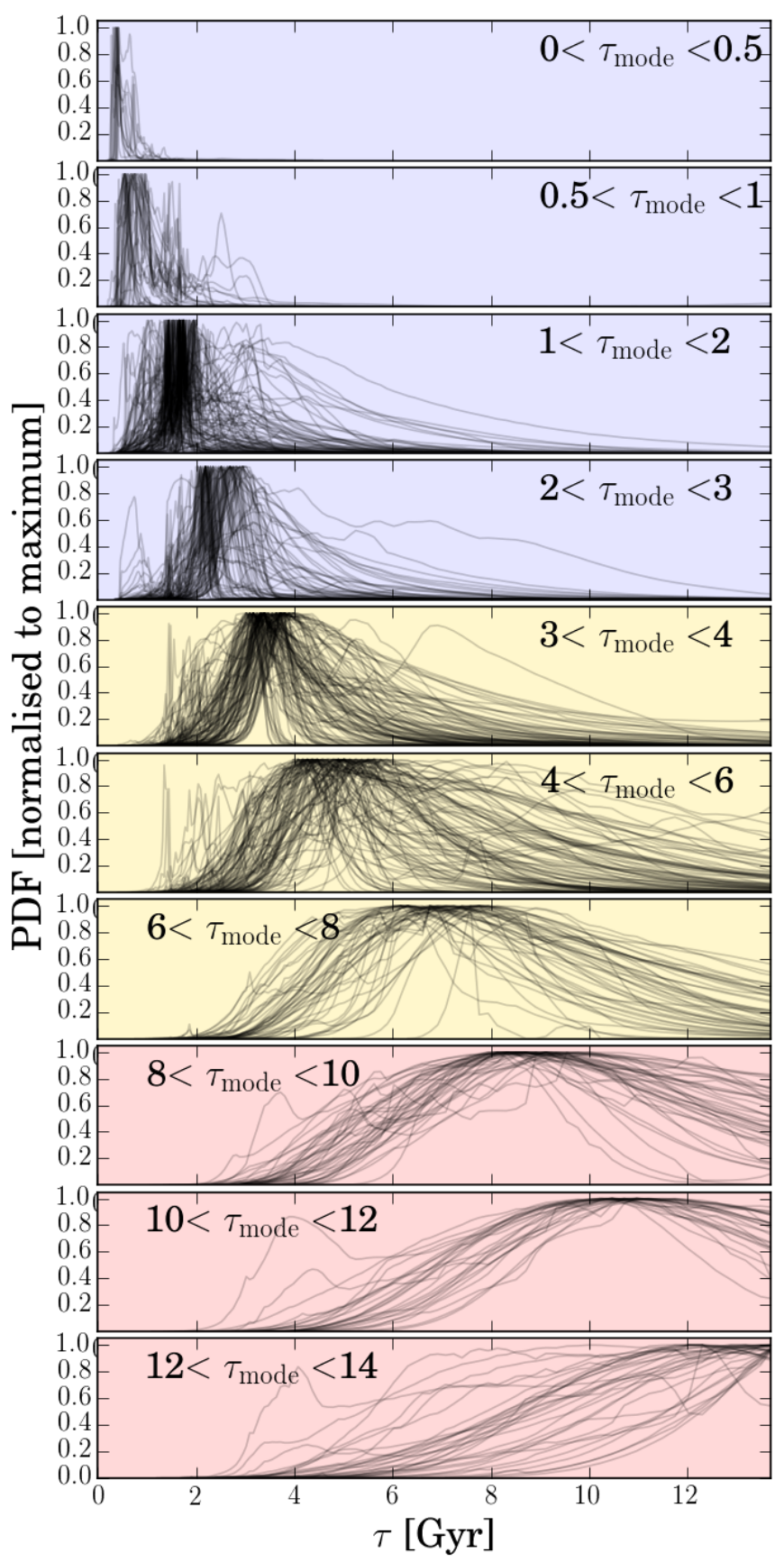

Fig. 7. Diversity of the computed age PDFs: All age PDFs of the CoRoGEE sample, grouped in bins of mode age. Background colours correspond to the three age bins used in Fig. 14.

\subsubsection{Systematic uncertainties}

For population studies of red giants, there are three main sources of systematic age uncertainties:

1. The accuracy of seismic masses: an important source of age bias comes from possible systematic errors in mass, which are likely to be small $(<10 \%)$, but are very hard to quantify given that only a few objects or stars in clusters have masses known to within $10 \%$ or better. Because hard constraints on the accuracy of the seismic masses have started to appear only very recently (e.g. Miglio et al. 2016), we refrain from a quantitative analysis in this paper. Future analyses will use a revised version of the $\Delta v$ scaling relation. 




Fig. 8. Seismo-spectroscopic Hertzsprung-Russell diagram of the CoRoGEE stars, with the symbols indicating the qualitative behaviour of the age PDFs, as described in the legend.

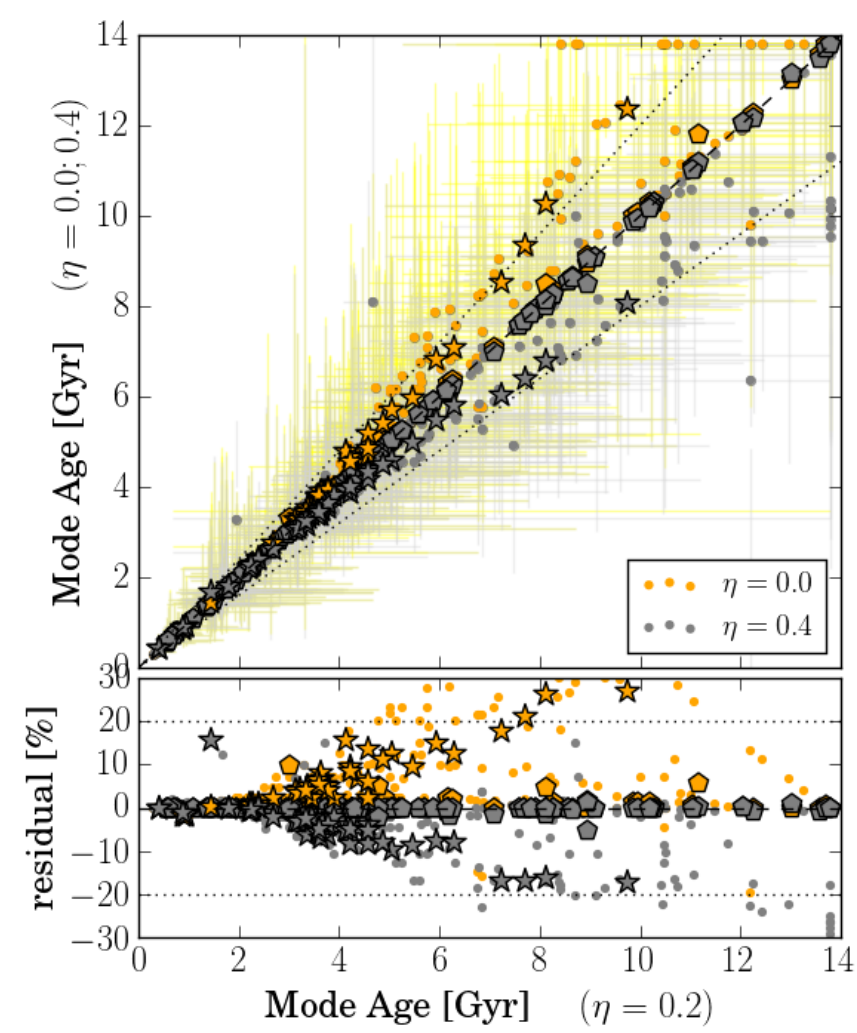

Fig. 9. Effect of non-canonical mass-loss assumptions on our derived ages. The upper panel shows the ages derived using a non-canonical mass-loss parameter $\eta_{\text {Reimers }}=0.0(0.4)$ in orange (grey), while the lower panel zooms into the differences. Again, known RC stars are plotted as stars, RGB stars as pentagons.

2. Mass loss: the accuracy of age-mass relations for red giants relies on our incomplete knowledge of stellar physics. While a relatively simple mass-age relation is expected for RGB stars, the situation for RC or early AGB stars is different: if these stars undergo a significant mass loss near the tip of the RGB, then the mass-age relation is not unique (for a given composition and input physics), since the mass observed at the RC or early-AGB stage may differ from the initial one (for a review see e.g. Catelan 2009; Miglio 2012) ${ }^{10}$. In the PARSEC isochrones, mass loss is included following the prescription of Reimers (1975). Figure 9 demonstrates the effect of varying our canonical value of the mass-loss efficiency $\eta=0.2$ to extreme values ( 0 or 0.4 , respectively). Our overall results are similar to the findings of Casagrande et al. (2016) for the SAGA sample: The impact of mass-loss on the age uncertainty increases with evolutionary stage, in the sense that RGB stars (especially seismically confirmed RGB stars) are almost unaffected by changes in $\eta$, while for RC stars we can change the age by up to $\pm 30 \%$ in some cases. However, for the vast majority of our stars the age uncertainty due to mass loss is $\lesssim 20 \%$.

3. Other input physics: it is well-known that the stellar physics input of theoretical isochrones (e.g. reaction rates, opacities, rotation, diffusion, He abundance, mass loss, or core overshooting) significantly affect the age and luminosities of the predicted stellar models at a given mass (e.g. Miglio et al. 2015; Noels \& Bragaglia 2015). At this time, the quantitative effects of each of the adopted input physics parameters on the isochrones are known in some detail through asteroseismology (e.g. Montalbán et al. 2013; Broomhall et al. 2014; Lebreton \& Goupil 2014). However, a real calibration of stellar models through seismology has only started recently ${ }^{11}$. A detailed comparison of the available stellar models has not yet been performed, but a recent study (Miglio et al., in prep.) suggests that the age spread models computed with different stellar evolution codes for an early AGB star at solar metallicity is around $7 \%$ for a $1 M_{\odot}$ star, $11 \%$ for a $1.5 M_{\odot}$ star, and $25 \%$ for a $2 M_{\odot}$ star. For this paper, we extrapolated these values to the full mass range, and neglected any possible dependency on metallicity.

We can now define our total age uncertainty as the quadratic sum of the (asymmetric) formal $1 \sigma$ uncertainty coming from PARAM, the uncertainty derived from the mass-loss test (Fig. 9), and the mass-dependent uncertainty coming from the comparison of different evolutionary codes.

Figure 10 displays the distribution of these total $1 \sigma$ age uncertainties as a function of age, colour-coded by field. The plot shows some important features:

- For stars between 4 and 10 Gyr, we observe an overall linear relation between age uncertainty and age. Because the finite age of the Universe (taken here as $\tau_{\max }=13.8 \mathrm{Gyr}$ ) is included in the age prior (which is flat in $\log \tau$ ), the methodintrinsic age uncertainties reach a maximum at $\tau=9 \mathrm{Gyr}$ and decrease again towards greater ages.

- A sizeable number of stars have a PDF maximum at the age limit: Most of these objects can be safely assumed to be old thick-disc stars.

- In the younger regime, we see a complicated behaviour in the age uncertainty-age diagram: Some stars appear to cluster around certain age values. These do not correspond to the grid points of our PARSEC models (which is much finer: $\Delta \log (\tau[\mathrm{yr}])=0.01)$. The observed dip in the LRc01 age distribution is not statistically significant.

${ }^{10}$ In this context, the characterisation of populations of giants benefits greatly from estimates of the period spacings of the observed gravity modes, which allows a clear distinction to be made between RGB and RC stars (Bedding et al. 2011), and early-AGB stars (Montalbán \& Noels 2013).

${ }^{11}$ For example, it has become possible to determine the amount of convective-core overshooting during the main-sequence phase (Silva Aguirre et al. 2013; Deheuvels 2015). 


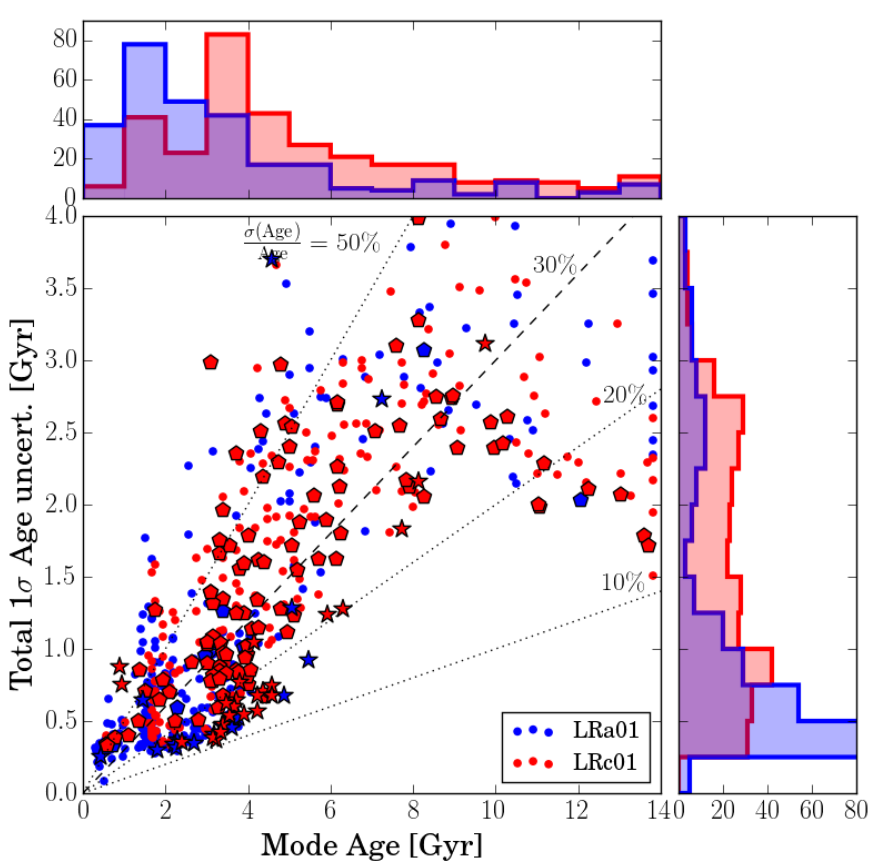

Fig. 10. Total age uncertainties as a function of age (or more precisely, the mode of the age PDF), for stars in LRc01 (red) and LRa01 (blue). Known RC stars are plotted as stars, RGB stars as pentagons. The black lines indicate lines of constant fractional age uncertainties (from left to right: $50 \%, 30 \%, 20 \%, 10 \%)$. The histograms in the top and right panels show the distribution of ages and age uncertainties, respectively.

- Although the age uncertainties are certainly non-negligible, the top panel of Fig. 10 suggests the indirect result of Miglio et al. (2013b), who used stellar population synthesis models to conclude that the stars in LRa01 are typically younger than the LRc01 population. To make this statement more quantitative, the histograms have to be corrected for selection effects, as we discuss in Sect. 4.2 below.

\subsubsection{Estimating age errors from simulated stars}

As an additional check of our age estimates, we opted to simulate the CoRoGEE sample based on the chemodynamical model of Minchev et al. $(2013,2014 a, M C M)^{12}$. The final snapshot of the MCM galaxy consists of $953206 N$-body particles with age, chemical, and kinematic information. To translate these mass particles into simulated stars, Piffl (2013) first used the MCM model as an input for the Galaxia code (Sharma et al. 2011) in the context of a simulated RAVE survey. Here, we used the same code to simulate a CoRoGEE-like sample from the MCM galaxy. A detailed description of the chemodynamical mock is given in Anders et al. (2016b). In the following, we briefly summarise the procedure.

We first simulated the stellar populations in the CoroT fields and calculated observed magnitudes for these mock stars using the new PanSTARRS-1 3D extinction map of Green et al. (2015) as our Galactic extinction model. In the next step, we applied the effective CoRoGEE selection function (assuming that it only depends on $H$ and $J-K_{\mathrm{s}}$ ) by selecting stars randomly from small boxes in the colour-magnitude diagram (see Fig. 4

\footnotetext{
12 The results are largely independent of the model used. However, we note that the MCM model is a thin-disc model only, and therefore does not include stars older than $11.7 \mathrm{Gyr}$.
}
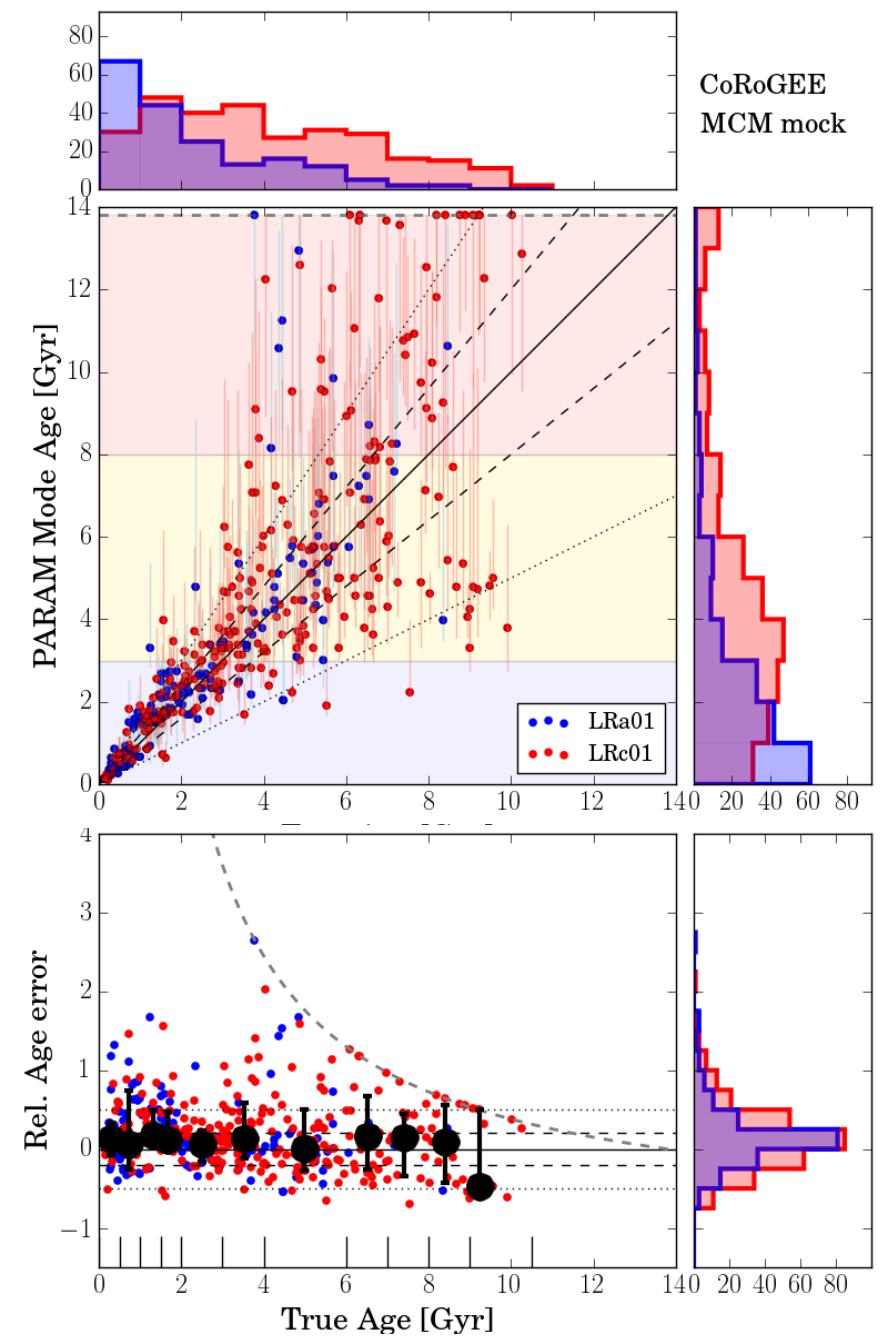

Fig. 11. Estimating systematics of our age estimates using simulated stars. The scatter plot in the upper panel shows estimated PARAM ages of the CoRoGEE mock stars (and their statistical $1 \sigma$ uncertainties as error bars) vs. the true ages of the parent $N$-body particle. The histograms to the sides of this plot show the corresponding age distributions of the two CoRoT fields. The background colours correspond to the three age bins used in Sect. 4. The lower plot shows the relative age error $\frac{\tau_{\text {PARAM }}-\tau_{\text {true }}}{\tau_{\text {true }}}$ as a function of the true age. The black symbols correspond to the median age error in each age bin indicated on the $x$ axis. The various lines correspond to a one-to-one relation, $20 \%$ and $50 \%$ deviation, and the age boundary at $13.8 \mathrm{Gyr}$.

of Anders et al. 2016b). While this is certainly a simplification of the true CoRoGEE selection (see Sect. 2), it was the only way in which our forward model could be realised. We also simulated Gaussian observational errors in the stellar parameters $T_{\text {eff }}, \Delta v, v_{\max },[\mathrm{Z} / \mathrm{H}]$ and magnitudes, and then ran the Bayesian parameter estimation code PARAM, exactly as was done with the real data.

Using this simulation, we can now address the question of how well our recovered PARAM age estimates correspond to the true stellar ages given by the model: The upper panel of Fig. 11 shows estimated vs. true ages, the lower panel presents the relative age error $\frac{\tau_{\text {PARAM }}-\tau_{\text {true }}}{\tau_{\text {true }}}$ as a function of the true age. The black symbols correspond to the median age error in each age bin indicated on the $x$ axis, demonstrating that our method tends to systematically overestimate the true ages by around $10-15 \%$, with the scatter increasing towards greater ages. A small systematic 
shift is expected, as the Galaxia input isochrones (Padova; Marigo et al. 2008) are slightly different from those used by PARAM (PARSEC; Bressan et al. 2012). The histograms to the sides of the top plot show how the true age distributions (in the model) of the two CoRoT fields are distorted by the measurement procedures.

As is clear from Fig. 11 and as shown in the previous section, our derived age estimates should be treated with caution, and considered relative age indicators rather than unbiased absolute age estimates. Therefore, in this paper we only use the age information to separate our stars into three wide age bins: Stars with derived PARAM ages younger than 3 Gyr ("young"), stars with PARAM ages between 3 and 8 Gyr ("intermediate"), and stars measured to be older than 8 Gyr ("old"). The typical forms of age PDFs for stars in these three bins are shown in Fig. 7 (coloured panels). The same coloured regions in the top panel of Fig. 11 can be used to assess the contamination in each of the three age bins. In summary, the simulation suggests that the contamination by old stars in the young bin and the contamination by young stars in the old bin are negligible.

\subsection{Distances and extinctions}

As in Rodrigues et al. (2014), distances and extinctions were calculated by comparing the previously derived absolute magnitude with the observed magnitudes in several passbands (see Sect. 2.3), assuming a single extinction curve (Cardelli et al. 1989; O'Donnell 1994), using the bolometric corrections of Marigo et al. (2008) and the corresponding extinction coefficients (Girardi et al. 2008). Because PARAM uses photometric measurements from many filters over a wide wavelength range (see Sect. 2.3), our distance uncertainties are much smaller than the uncertainties expected from the distance-radius relation (as adopted in e.g. Miglio et al. 2013b). For more details, we refer to Sect. 3 of Rodrigues et al. (2014), and to Appendix A.

We carried out comparisons with extinction estimates from the literature in Appendix A.3, finding that our precise extinction values are best matched by the spectro-photometric method developed in Schultheis et al. (2014).

\subsection{Kinematics}

The 6D phase-space coordinates, along with their uncertainties are available for a subset of 504 stars. For this subset, orbital parameters were computed in the same manner as in Anders et al. (2014). Most of the more distant stars, however, still have too large proper motion uncertainties $\left(>50 \mathrm{~km} \mathrm{~s}^{-1}\right.$ in the tangential component of the space velocity, $v_{\mathrm{T}}$ ) to be useful even for statistical kinematic studies, as our sample is too small to allow for good statistics in the presence of noisy kinematical data (see Table 1). When examining the kinematical properties of our sample, we therefore concentrated on the most reliable parameters whenever possible.

One relatively robust parameter is the guiding-centre radius of a stellar orbit, which we computed using the approximation $R_{\text {guide }}=\frac{L_{z}}{v_{\mathrm{c}}}=\frac{v_{\phi} \cdot R_{\mathrm{Gal}}}{v_{\mathrm{c}}}$ (e.g. Casagrande et al. 2011). Here, $L_{z}$ denotes the angular momentum, $v_{\phi}$ the $\phi$-component of the space velocity, and $v_{\mathrm{c}} \approx 220 \mathrm{~km} \mathrm{~s}^{-1}$ the circular velocity at the star's position - which for for our purposes can be assumed to be approximately constant over the Galactocentric distance range considered.


Fig. 12. Location of the CoRoT-APOGEE stars in Galactocentric Cartesian coordinates $\left(X_{\mathrm{Gal}}, Y_{\mathrm{Gal}}-\right.$ top panel $)$ and cylindrical coordinates $\left(R_{\mathrm{Gal}}, Z_{\mathrm{Gal}}-\right.$ bottom $)$. Blue dots correspond to LRa01 targets, red dots to LRc01 targets. The APOGEE DR10 high-quality giant sample (Anders et al. 2014) is shown in the background (black dots).

\section{The $[\alpha / \mathrm{Fe}]-[\mathrm{Fe} / \mathrm{H}]$-age diagram at different Galactocentric distances}

In addition to the presentation of the CoRoT-APOGEE data in the past two sections, the goal of this and following work is to study the age-abundance-kinematics relationships of the Milky Way disc outside the solar cylinder. To illustrate the value of our sample for Galactic archaeology, in this section we study the $[\alpha / \mathrm{Fe}]-v s .-[\mathrm{Fe} / \mathrm{H}]$ abundance relationship with Galactocentric distance and age.

The CoRoGEE sample has the novel advantage of covering a wide radial range of the Galactic disc $\left(4 \mathrm{kpc}<R_{\mathrm{Gal}}<14 \mathrm{kpc}\right)$ with red giants for which both asteroseismic and high-resolution spectroscopic data are available. Our final sample comprises 606 stars with converged stellar parameters and distances in the two CoRoT fields LRa01 and LRc01. However, given the extended radial and age baselines, this sample size forces us to constrain our analysis to broad bins of Galactocentric distances and ages instead of using full distribution functions. Moreover, we recall that systematic uncertainties probably affect the estimated ages presented here. Hence, we focus our analysis on larger age bins.

Following the path of Chiappini et al. (2015), we now examine the $[\mathrm{Fe} / \mathrm{H}]-[\alpha / \mathrm{Fe}]$-age space also outside the solar neighbourhood, analysing the CoRoGEE stars for which we now also have age information. We compare our findings to the predictions of 


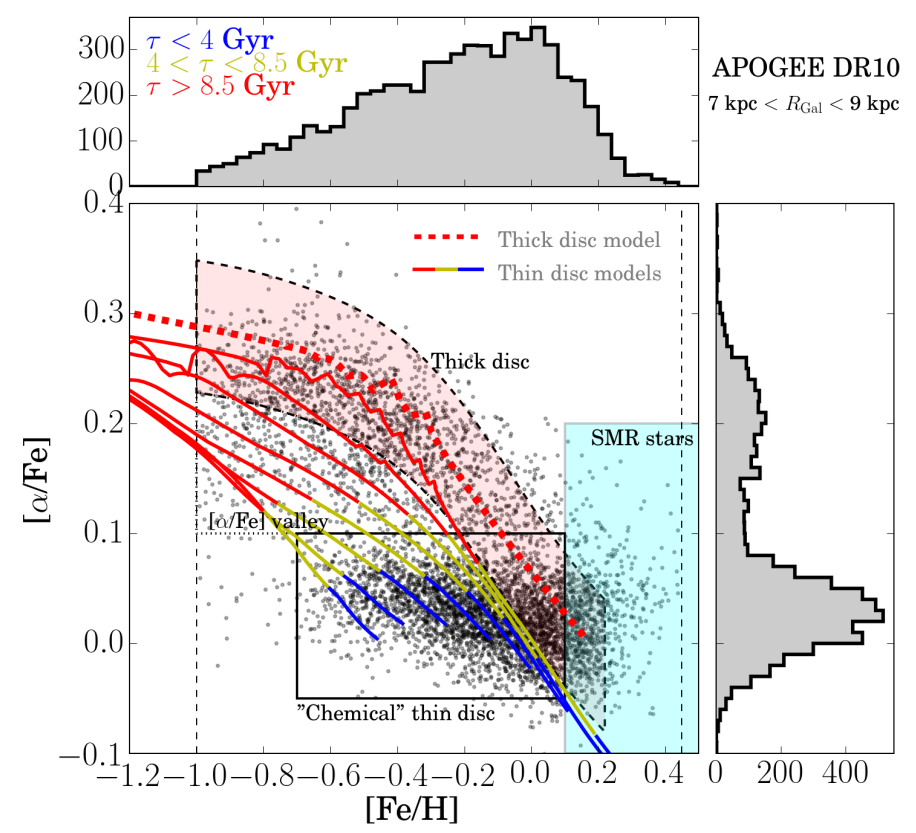

Fig. 13. The $[\alpha / \mathrm{Fe}]$ vs. $[\mathrm{Fe} / \mathrm{H}]$ diagram of the APOGEE DR 10 highquality giant sample (Anders et al. 2014; grey dots) in the range $7 \mathrm{kpc}<$ $R_{\text {Gal }}<9 \mathrm{kpc}$. Overplotted with colours are the thin- and thick-disc chemical-evolution models of Chiappini (2009): the solid lines correspond to the chemical tracks of the thin disc at different Galactocentric annuli (from left to right: $18 \mathrm{kpc}, 16 \mathrm{kpc}, 14 \mathrm{kpc}, 12 \mathrm{kpc}, 10 \mathrm{kpc}, 8 \mathrm{kpc}$, $6 \mathrm{kpc}, 4 \mathrm{kpc}$ ). The colours indicate the age (or look-back time), as indicated in the top panel. The dashed line represents a thick-disc model (for $R_{\mathrm{Gal}}=6 \mathrm{kpc}-$ the Galactocentric dependency for the thick-disc models computed in Chiappini (2009) are minor - see text for more details).

chemical-evolution models, as well as to recent chemodynamics results.

\subsection{Understanding $[\alpha / \mathrm{Fe}]$ vs. $[\mathrm{Fe} / \mathrm{H}]$ diagrams with a chemical-evolution model}

$[\mathrm{X} / \mathrm{Fe}]$ vs. $[\mathrm{Fe} / \mathrm{H}]$ diagrams, and in particular the $[\alpha / \mathrm{Fe}]$ vs. $[\mathrm{Fe} / \mathrm{H}]$ diagram, are widely used diagnostic tools to constrain the enrichment history of stellar populations. High-resolution spectroscopic data reveal two clearly-separated disc components (thin and thick) in the $[\alpha / \mathrm{Fe}]$ vs. $[\mathrm{Fe} / \mathrm{H}]$ diagram, which follow their own age-metallicity relations (e.g. Gratton et al. 1996; Fuhrmann 1998; Ramírez et al. 2007; Anders et al. 2014). The valley between the two sequences in this diagram can hardly be attributed to simple sample selection effects (Anders et al. 2014; Nidever et al. 2014) and is probably a real characteristic of the Galactic disc ${ }^{13}$, as we discuss below.

As a starting point, in Fig. 13 we compare the bulk of APOGEE DR10 data analysed in Anders et al. (2014) with the predictions of the set of Galactic chemical-evolution models of Chiappini (2009). The figure shows the location of Anders et al. (2014) high-quality disc sample in the $[\alpha / \mathrm{Fe}]$ vs. $[\mathrm{Fe} / \mathrm{H}]$ diagram, together with the histograms of these parameters. Overplotted are the chemical-evolution tracks of Chiappini (2009) for various bins in Galactocentric distance, colour-coded by age.

The thin-disc models shown in Fig. 13 were obtained by varying the accretion timescale onto the disc, assuming it to be

\footnotetext{
${ }^{13}$ However, see Bovy et al. (2012) for a different explanation.
}

shorter in the inner regions and longer in the outer parts (typical for MW chemical-evolution models with inside-out formation; e.g. Chiappini et al. 1997, 2001; Hou et al. 2000). For this reason, the thin disc at the solar vicinity formed on a longer timescale than the thick disc, and towards the inner disc regions the infall timescales of both components approach each other (but there is still a difference in the star-formation efficiency). This explains why the thin-disc model curve at $4 \mathrm{kpc}$ is close to the thick disc curve (see Fig. 14 in the next section), but reaches a lower $[\mathrm{Fe} / \mathrm{H}]$ value. The details of the thin-disc model can be found in Minchev et al. (2013, Sect. 3).

From a pure chemical-evolution point of view, the thick disc can be modelled as a separate Galactic component with high starformation efficiency and a short infall timescale. Such a model naturally predicts a population of mostly old $[\alpha / \mathrm{Fe}]$-enhanced stars with a metallicity distribution peaking around -0.5 dex (e.g. Soubiran 1999) and explains some of the abundance patterns observed in high-resolution solar-vicinity samples that are classified as thick-disc-like (Chiappini 2009). When building a chemical-evolution thick-disc model of this type, one has considerable freedom in the choice of parameters because tight observational constraints are still lacking. As an example, for the thick disc models one can assume that its formation is completed within 2-3 Gyr (in order to obtain a population that is mostly older than $10 \mathrm{Gyr}$ ), but there is no tight constraint on the tail of the age distribution. While thin-disc models have to reproduce the chemical-abundance patterns at the present time in the local interstellar medium, the final metallicity and abundance pattern for the thick disc is still under debate (solar or super-solar depending on how this component is defined in the different datasets). Therefore, the thick-disc curve illustrated by the dashed line in Fig. 13 could be easily extended to higher metallicities, whereas the same is not true for the thin-disc curves (especially for the one at the solar-vicinity position).

Figure 13 shows that these chemical models broadly agree with the two main features of the Galactic disc $[\alpha / \mathrm{Fe}]$ vs. $[\mathrm{Fe} / \mathrm{H}]$ diagram: the location of the bulk of thin-disc stars at $[\alpha / \mathrm{Fe}]<0.1$ and $[\mathrm{Fe} / \mathrm{H}]>-0.8$ (rectangular box labelled "chemical" thin disc in the figure), and the position of the stars following a thick-disc track (marked by the rose-coloured region and the thick red dashed line). Within the framework of these models, the thin-disc sequence can be explained as a mixture of relatively young (age $\lesssim 5$ Gyr) stars, originating from different birth regions within the Galactic disc that have had different enrichment histories. In contrast, for the thick disc the metallicity distribution peaks at $\sim-0.5$ (e.g. Rocha-Pinto \& Maciel 1996; Kotoneva et al. 2002; Nordström et al. 2004; Holmberg et al. 2007), and a large number of stars is expected at high $[\alpha / \mathrm{Fe}] \mathrm{ra}-$ tios and metallcities below $\sim-0.2$. Because of the co-existence of thick and thin disc in this diagram, a gap or dip in the $[\alpha / \mathrm{Fe}]$ vs. $[\mathrm{Fe} / \mathrm{H}]$ diagram should thus be naturally produced.

Of course, the exact absolute position of the tracks with respect to the data depends not only on the calibration zeropoint of the APOGEE abundances ${ }^{14}$, but also on the choice of stellar yields, IMF, and star-formation efficiency. As shown in Chiappini (2009), these models provide a good description of the observed shifts of several abundance ratios as a function of

${ }^{14}$ As an example, from SDSS DR10 to DR12, there has been a shift of $\sim 0.1$ dex in the calibrated metallicities (Holtzman et al. 2015; Martig et al. 2015), and further improvements might affect the metallicity scale at the same level. A +0.05 dex shift in $[\alpha / \mathrm{Fe}]$ is also observed when moving from DR10 to DR12. As the same shift is observed between a Gaia-ESO Survey sample and the DR12 values, we opted to retain the DR10 values for the comparison in Fig. 13. 
metallicity for the solar radial bin, once the thick and thin discs are defined via kinematics (as in Bensby et al. 2003).

The reason we present a comparison with a model computed before the data in Fig. 13 were available is to illustrate how the predictions of a pure chemical-evolution model that was in agreement with chemical abundances (among other observables) in the local volume performs when compared to the new samples of stars now covering larger portions of the disc. Clearly, one of our near-term goals is to further explore the parameter space (especially new constraints on the stellar yields and their metallicity dependency) of these models and identify those that best fit the new observational constraints. However, the main challenges to the interpretation of discrete thin and thick discs (as modelled in Chiappini et al. 1997; Chiappini 2009) are on the one hand the existence of so-called super-metal-rich (SMR) stars (Grenon 1972; Trevisan et al. 2011) in the solar neighbourhood ${ }^{15}$, and on the other hand the fact that not all thin-disc stars with metallicities below -0.2 can be explained as high-eccentricity intruders from outer regions (Anders et al. 2014).

The existence of SMR stars is commonly attributed to a significant radial mixing of stellar populations within the Galactic disc (e.g. Grenon 1989, 1999; Chiappini 2009; Kordopatis et al. 2015). In agreement with previous studies, Kordopatis et al. (2015) conclude that SMR stars in the solar neighbourhood must have migrated from far inside the solar annulus. Recently, Schönrich \& Binney (2009), Brunetti et al. (2011), Minchev et al. (2013, 2014b) and Kubryk et al. (2015a,b) have argued that chemical-evolution models for the Milky Way cannot be viewed independently of its dynamical evolution, and found different prescriptions for the merging of these two aspects of Galactic evolution. In the next subsection we separate the $[\alpha / \mathrm{Fe}]-$ $[\mathrm{Fe} / \mathrm{H}]$ diagram into bins of age and Galactocentric distance and compare our data to a chemical-evolution model. This is useful because the latter form the backbone of many recent chemodynamical approaches.

\subsubsection{Binning the data in Galactocentric distance and age}

While the division of the massive APOGEE dataset into various Galactic zones has been the subject of previous investigations (Anders et al. 2014; Hayden et al. 2014; Nidever et al. 2014; Hayden et al. 2015), we now can make use of the unique seismic information from CoRoT to show, for the first time, $[\alpha / \mathrm{Fe}]-[\mathrm{Fe} / \mathrm{H}]$-age diagrams, outside the HIPPARCOS volume, in several Galactocentric bins.

Figure 14 presents one of the main results of this paper: the $[\alpha / \mathrm{Fe}]$ vs. $[\mathrm{Fe} / \mathrm{H}]$ diagram for the CoRoGEE sample, split into five bins of Galactocentric distance, as indicated in each panel.

As in Fig. 13, we include in Fig. 14 the $[\alpha / \mathrm{Fe}]$ vs. $[\mathrm{Fe} / \mathrm{H}]$ model tracks of Chiappini (2009). Figure 15 has the same format as Fig. 14, only that the data are now binned in guiding-center radius $R_{\text {guide }}$ instead of Galactocentric distance, to mitigate the effect of stellar mixing by "blurring" (Schönrich \& Binney 2009). In this plot, the size and transparency of the symbols encode the uncertainty in both stellar age and guiding-center radius, because both quantities may have considerable uncertainties. The interpretation of these figures is difficult because of the low statistics

\footnotetext{
15 SMR stars are defined as stars whose metal abundance exceeds the metallicity of the local present-day interstellar medium. This value is dependent on Galactocentric distance and is constrained by the presentday abundance gradient in the interstellar medium. For the solar vicinity, SMR stars are found in the region illustrated by the blue rectangular box in Fig. 13.
}

and the noise arising from proper motion uncertainties and radial migration. We analyse the two figures simultaneously below.

The main results we derive from these figures are:

- The shift of the peak of the thin-disc metallicity distribution function from higher to lower metallicities as one moves towards larger Galactocentric distances (Anders et al. 2014; Hayden et al. 2015) is accompanied by a dominance of younger ages towards the outermost radial bins. However, the exact relative number of young and old stars in each radius bin can be slightly biased as a consequence of the detectability of oscillations: younger stars are on average more luminous, and therefore exhibit larger oscillation amplitudes that are easier to detect at large distances.

- While the inner Galaxy is dominated by stars with thick-disclike chemistry (elevated $[\alpha / \mathrm{Fe}]$ ratios) with a large number of old stars (but see below), almost no high- $[\alpha / \mathrm{Fe}]$-old stars are found in the outermost radial bin. This result is believed to be a manifestation of the shorter scale length of the thick disc with respect to the thin disc (Bensby et al. 2011; Bovy et al. 2012; Cheng et al. 2012).

- A greater number of young-[alpha/Fe]-rich stars is seen in the two innermost bins (Chiappini et al. 2015). The existence of these stars strongly disagrees with the predictions of chemical-evolution models and is also impossible to explain by radial migration. For a discussion of the origin of these stars see Chiappini et al. (2015), Jofré et al. (2016), Yong et al. (2016).

- Surprisingly, the thin-disc chemical-evolution model adopted here provides a fairly good description of the main abundance ratio trends shown in the figures (especially in the outer parts of the Galaxy), both in terms of the abundance trends and in terms of expected dominant age. In particular, when guiding radii are used instead of the current Galactocentric distances, the agreement with the models is improved (see the 5-6 kpc and 6-7 kpc $R_{\text {guide }}$ bins). However, there is a clear disagreement above solar metallicity in all panels. Although part of the discrepancy might be attributed to uncertainties related to stellar yields ${ }^{16}$, it is tempting to interpret this result as a sign of radial migration, at least for the old and intermediate-age stars (see MCM13, Fig. 8).

- Interestingly, in each bin, stars with high $[\alpha / \mathrm{Fe}]$ abundances, regardless of their age, show a tendency to lie close to the

${ }^{16}$ Currently there are several uncertainties affecting the stellar yields of the different $\alpha$-elements. For core-collapse supernovae, few models were computed for metallicities above solar; moreover, most supernovae models tend to underestimate the ${ }^{24} \mathrm{Mg}$ yields. Other elements, such as $\mathrm{Ca}, \mathrm{Si}$, and $\mathrm{S}$, can have some contribution of SNIa as well. Even more importantly, the Galactic SNIa rate is still very uncertain (e.g. Matteucci \& Romano 1999; Mannucci et al. 2006). Although the thin disc model presented here reproduces the present SNIa rate at the solar vicinity well, overestimated SNIa rates at earlier times and/or at other Galactocentric distances cannot be excluded. In the inside-out thin-disc formation model, one of the assumptions is that the star-formation efficiency increases towards the inner regions. This feature was mainly constrained by the abundance gradients at present time. However, if the SNIa rate is overestimated, one would require lower star-formation efficiencies to reach the same final metallicity. The abundance ratios at the different Galactocentric distances can further constrain these models, because a larger star-formation efficiency would also predict larger $[\alpha / \mathrm{Fe}]$ ratios at larger metallicities. It is thus possible that, by exploring the parameter space of stellar yields and SNIa rates, one can obtain a better fit to the data shown in the Figure, but this is beyond the scope of the present work. 

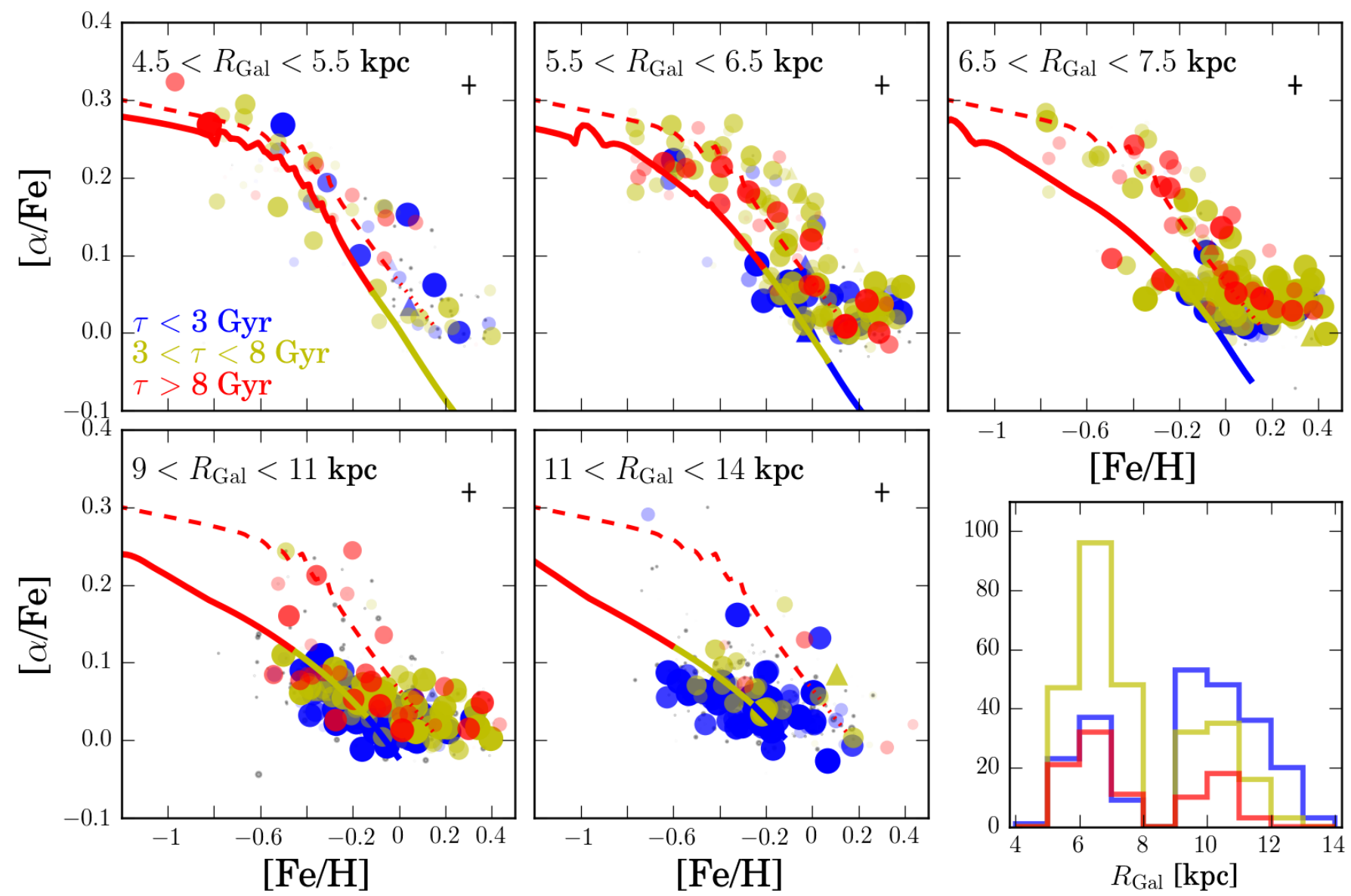

Fig. 14. The $[\alpha / \mathrm{Fe}]$-vs.- $[\mathrm{Fe} / \mathrm{H}]$ chemical plane for five different bins in Galactocentric distance $R_{\mathrm{Gal}}$. The colour represents our stellar age estimates, as indicated in the first panel: blue indicates stars younger than $3 \mathrm{Gyr}$, red stars older than $8 \mathrm{Gyr}$, and yellow intermediate ages. The point size and transparency of each data point encode the age uncertainty, i.e., a smaller and more transparent symbol corresponds to a lower probability to belong to the particular age bin. The few triangles correspond to stars whose measured radial velocity scatter is greater than $800 \mathrm{~m} / \mathrm{s}$ and which could be binaries. In the background of each panel, stars from the APOGEE DR12 main sample observed in similar Galactic regions are plotted as grey dots for comparison. The error bar in the upper right corner of each panel represents the typical (internal) uncertainty of the chemical abundances. The solid lines correspond to the thin-disc chemical-evolution model of Chiappini (2009) for different Galactocentric distances, and the dashed lines correspond to a thick-disc model at $R_{\mathrm{Gal}}=6 \mathrm{kpc}$. The lower right panel displays the overall $R_{\mathrm{Gal}}$ distributions of our sample split into the three age bins.

thick-disc curve. As the same thick-disc curve is shown in all panels, this result agrees with the relative constancy of the "high- $[\alpha / \mathrm{Fe}]$ sequence" discussed in Nidever et al. (2014). It is clear from this comparison that these stars can either be explained as being part of the thick disc, or as migrators coming from the inner radii (the thick disc curve is similar to the that for $R_{\mathrm{Gal}}=4 \mathrm{kpc}$, except for its higher star-formation efficiency, which leads to the appearance of $[\alpha / \mathrm{Fe}]$-enhanced stars at higher metallicities). These oldest metal-rich, $[\alpha / \mathrm{Fe}]-$ enhanced stars also resemble Galactic bulge stars in chemistry, so that radial migration from the bulge cannot be excluded as one possible interpretation.

- SMR stars are present even in the two outermost $R_{\mathrm{Gal}} / R_{\text {guide }}$ bins studied here (Anders et al. 2014); they comprise stars of all ages, in agreement with what was found by Trevisan et al. (2011) for solar-vicinity SMR stars. As explained previously, the end of the thin-disc curves is constrained by the present abundance gradient, which amounts to around $-0.07 \mathrm{dex} / \mathrm{kpc}$ for Fe (e.g. Anders et al. (2014)) and references therein). While the excess of SMR stars is not a problem in the inner bins (where the thin-disc curve extends to higher metallicities), it demonstrates a clear discrepancy for the two outermost bins analysed here. From the comparison with the models it is clear that the chemistry of these SMR stars is compatible either with the thick-disc curve or with the thin disc at $R_{\mathrm{Gal}}=4 \mathrm{kpc}$. We note, however, that the $R_{\mathrm{Gal}}=4 \mathrm{kpc}$ curve predicts intermediate ages for stars above metallicities $\sim-0.02$, while there are clearly older SMR stars in all panels. This is an indication that these stars indeed migrated from $R_{\mathrm{Gal}}<4 \mathrm{kpc}$. Unfortunately, the form of the present-day abundance gradients in the innermost regions of the Galactic disc is still unknown (see Stasińska et al. 2012 for a discussion): a constraint that would shed more light on the origin of these stars.

\subsection{Comparison with a chemo-dynamical model}

As first shown in Minchev et al. (2013), when radial migration is taken into account in a chemodynamical model of the thin disc, the oldest stars in the simulation have properties similar to what we commonly identify as the thick disc (this result 

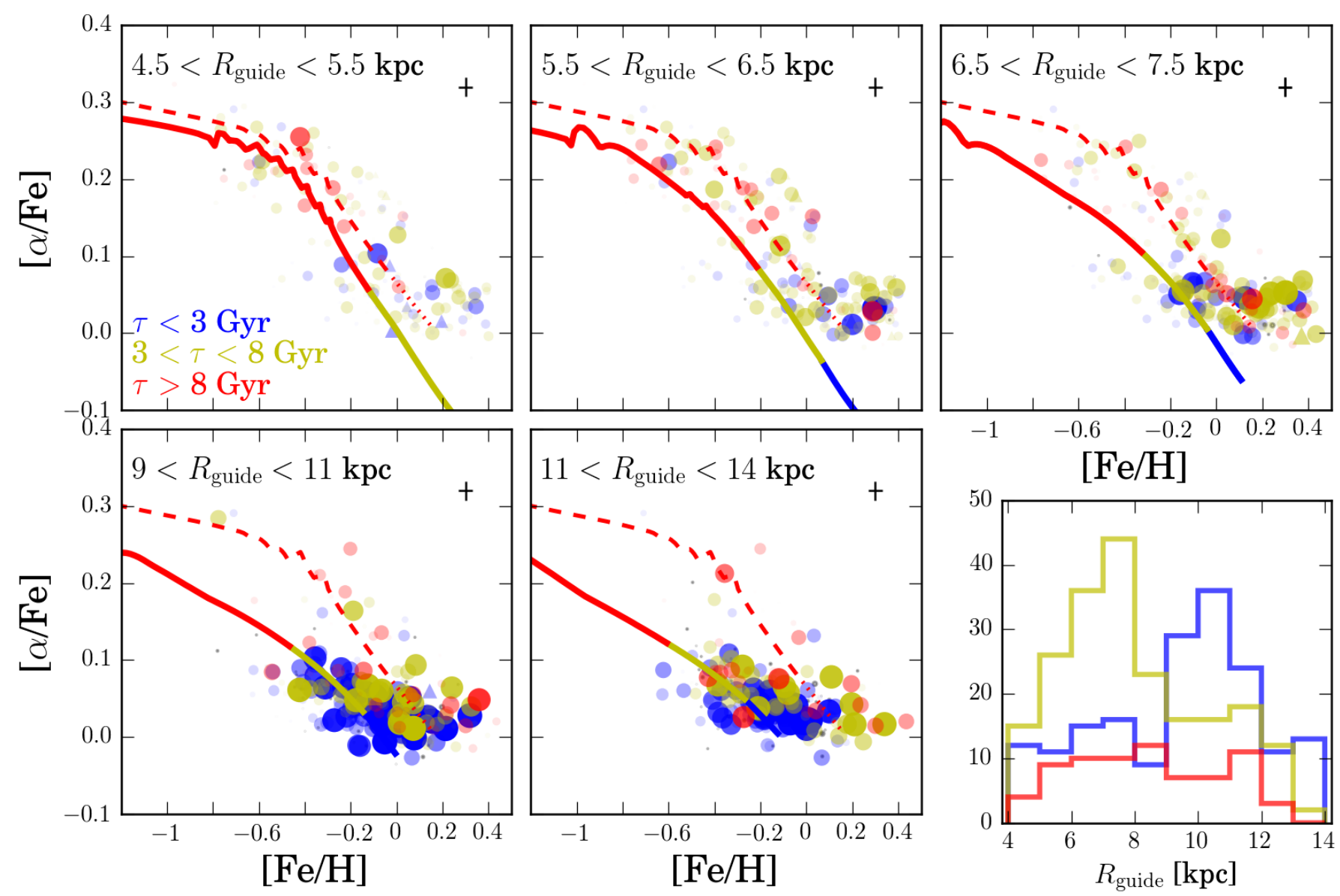

Fig. 15. Same format as Fig. 14, only that the data are now binned in guiding-center radius, $R_{\text {guide }}$, rather than Galactocentric distance, to mitigate the effect of stellar mixing by "blurring". Again, the colour-code represents the age, while the size and transparency now encode the uncertainty in stellar age and guiding-center radius. (If a star has a highly uncertain guiding radius - i.e. an $R_{\text {guide }}$ PDF which extends over multiple $R$ bins - it will appear as a faint dot in multiple panels of this figure.)

was later confirmed by Kubryk et al. $\left.2015 \mathrm{a}^{17}\right)$. Interestingly, although it is able to reproduce several properties of "the thick disc", our chemodynamical model does not predict a discontinuity in the $[\alpha / \mathrm{Fe}]$ vs. $[\mathrm{Fe} / \mathrm{H}]$ diagram $^{18}$. The reason for this discrepancy might be the existence of a discrete thick-disc component (Chiappini et al. 1997; Chiappini 2009), with its specific chemical pattern, which was not included in the MCM model. To solve this problem, a proper comparison between the MCM model predictions and observations is required. Because astronomical surveys are often affected by non-trivial selection effects, the comparison of survey catalogues with a Galactic model is much easier when a mock observation of the model is created (e.g. Binney \& Sanders 2016).

In this section we describe our selection of a CoRoGEElike sample from an $N$-body simulation, using the example of the chemodynamical $N$-body model analysed in Minchev et al.

\footnotetext{
${ }^{17}$ In this case, differently from Minchev et al. (2013), the authors followed a suggestion made in Brunetti et al. (2011):the radial migration process was approximated by a diffusion process with diffusion coefficients that varied in time and position. These were extracted from an $N$-body+SPH simulation of a galaxy very different from the Milky Way and implemented in a standard chemical-evolution model. The coefficients were then re-scaled to fit the local G-dwarf metallicity distribution.

${ }^{18}$ However, when selecting particles using the same kinematical criteria as in Bensby et al. (2003), it was possible to recover the two sequences in the $[\alpha / \mathrm{Fe}]$ vs. $[\mathrm{Fe} / \mathrm{H}]$ diagram.
}

(2013), Minchev, Chiappini, \& Martig (2014a, MCM). We have chosen two different paths to simulate the observations: 1. a "simple" mock in which we choose $N$-body particles such that we match the observed spatial distribution of our program stars (and simulating the red-giant age bias with a simple prior); and 2. a more sophisticated mock that used a modified version of the Galaxia synthetic stellar population code (Sharma et al. 2011; Piffl 2013), the new PanSTARRS-1 3D extinction map of Green et al. (2015), and a representation of the CoRoGEE selection function. The procedures leading to the two versions of mock observations are sketched in Fig. 16 and are explained in Anders et al. (2016b). As we show below, these two versions of an MCM-CoRoGEE mock sample each have their advantages and drawbacks. In summary, while the simple mock by construction matches the space distribution of the observed sample perfectly, the sophisticated mock recovers the observed age distribution very well (see Anders et al. 2016b).

Figure 17 shows the main result of our mock samples: each row contains the (observed or modelled) $[\alpha / \mathrm{Fe}]$ vs. $[\mathrm{Fe} / \mathrm{H}]$ diagrams in the same $R_{\mathrm{Gal}}$ and age bins as in Figs. 14 and 15, to facilitate a qualitative comparison with the data. We discuss the main results from Fig. 17 below.

1. Sophisticated mock: Anders et al. (2016b) have shown that the observed age distributions in the two CoRoGEE fields are very well recovered by the sophisticated mock. This is also seen in the second row of Fig. 17: the age mix of CoRoGEE 


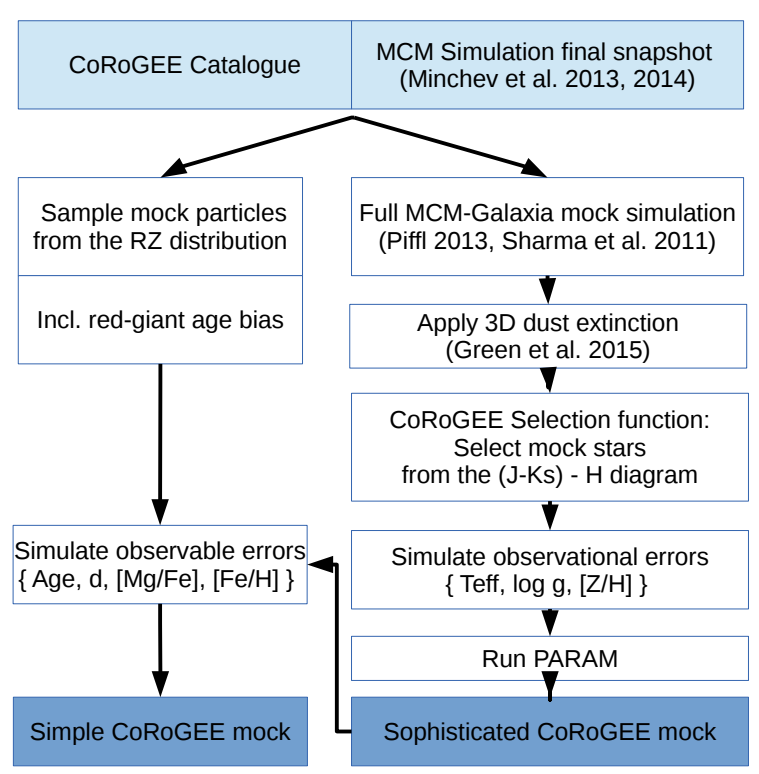

Fig. 16. Scheme illustrating how the CoRoGEE mock observations were obtained from the MCM model. The steps are explained in more detail in Anders et al. (2016b).

stars is better reproduced in the sophisticated mock than in the simple mock. However, the sophisticated mock obviously misses the distant and metal-poor $([\mathrm{Fe} / \mathrm{H}]<-0.5)$ stars that are present in the data. This indicates that our forward modelling of the sample selection is imperfect for various reasons: 1. a mismatch between the MCM-Galaxia model's starcounts with 2MASS in the CoRoT fields (Anders et al. 2016b), 2. a stronger extinction in the PanSTARRS extinction maps (Schlafly et al. 2014; Green et al. 2015) compared to the CoRoGEE data (see Appendix A.3), 3. a more complex selection function than $S \propto S$ (field, $H, J-K_{\mathrm{s}}$ ) (see Sect. 2.2), and 4. stochasticity due to the small sample size. We therefore refrain from interpreting the number counts in the sophisticated CoRoGEE mock, as we did not recover the overall distributions in the abundance diagrams.

2. Simple mock with true ages: by construction (selection of mock particles from the $R_{\mathrm{Gal}}-Z_{\mathrm{Gal}}$ plane), the simple mock matches the space distribution of the CoRoGEE sample perfectly. The simple mock also matches the observed metallicity distributions much better than the sophisticated mock. The plot also demonstrates that despite the quite strong radial mixing in the MCM model, there is little age mixing in each of the $[\alpha / \mathrm{Fe}]$ vs. $[\mathrm{Fe} / \mathrm{H}]$ diagrams. The age- $[\alpha / \mathrm{Fe}]$ relation of the input models is largely preserved, while the data show a significantly more complex situation. In concordance with the data, the density of the old $[\alpha / \mathrm{Fe}]$-enhanced thin disc (i.e. the "thick disc" in MCM) decreases towards outer regions. However, the data suggest that the $[\alpha / \mathrm{Fe}]$-enhanced component has a much broader age distribution than in the model. This result depends little on the functional form of the simulated age bias.

3. Effect of adding age errors: when we add realistic age errors using the PARAM results of the sophisticated mock (see Fig. 11 and Anders et al. 2016b), part of the age mixing in the $[\alpha / \mathrm{Fe}]$ vs. $[\mathrm{Fe} / \mathrm{H}]$ diagram can be explained by our measurement procedure. This is insufficient to explain the observed younger ages of many $[\alpha / \mathrm{Fe}]$-enhanced stars, however. In particular, our method-intrinsic age errors cannot explain the presence of young $[\alpha / \mathrm{Fe}]$-rich stars, while possible close-binary stellar evolution cannot explain the different abundance of these stars in the two CoRoT fields (see also Chiappini et al. 2015; Martig et al. 2015; Yong et al. 2016; Jofré et al. 2016).

4. SMR stars in the outer disc: as discussed in the previous section, the metal-rich stars in the two outer bins cannot be explained with the present chemical models. Since the MCM mocks also do not produce this metal-rich intermediateage population in the outer parts of the disc, either a much stronger radial migration than present in MCM is at work, or the thick disc star-formation history extends to greater ages (i.e., for longer than $2 \mathrm{Gyr}$ ). Another explanation might also be bulge stars ending up in the outer disc (Barbuy \& Grenon 1990); these were not included in the MCM simulation.

Our simple mock outperforms the sophisticated mock in almost all respects (except for the match with the overall age distributions). It highlights two important features in the data that are not reproduced by the MCM model: the broad observed age distribution of the $[\alpha / \mathrm{Fe}]$-enhanced sequence in the inner Galactic disc, and that more intermediate-age SMR stars are located in the outer disc than predicted.

\section{Conclusions}

In this first CoRoGEE paper, we have demonstrated the usefulness of combining asteroseismic and spectroscopic data in the framework of Galactic archaeology. Using global asteroseismic parameters $\Delta v$ and $v_{\max }$ determined from CoRoT light curves, together with atmospheric stellar parameters measured by SDSS-III/APOGEE and broad-band photometry, we have calculated masses, radii, ages, distances and extinctions for more than 600 red giants distributed over a large Galactocentric distance interval. In this section, we briefly summarise the main results of our work.

The relative statistical uncertainties in our primary derived quantities from the Bayesian model fitting performed by the PARAM code amount to $\lesssim 2 \%$ in distance, $0.08 \mathrm{mag}$ in $A_{V}, \sim 4 \%$ in radius, $\sim 9 \%$ in mass and $\sim 25 \%$ in age. In agreement with previous studies, we find that the individual age probability distributions can be complex in shape, suggesting that the age information needs to be used with some care, for example, by using wide age bins. Equally importantly, systematic uncertainties in the fundamental seismic parameters as well as in the comparison with stellar models may affect the absolute scale of our derived ages to some degree.

We provide a number of checks (surface gravity comparison, grid-based vs. scaling relation results, extinction maps) that demonstrate the overall reliability of our analysis for the use with statistical samples in Appendix A. The CoRoGEE sample enabled us to study for the first time the $[\alpha / \mathrm{Fe}]-[\mathrm{Fe} / \mathrm{H}]$-age relation beyond the solar vicinity. We separated the sample into large bins of age, guiding-centre radius, and Galactocentric distance, to study stellar populations in the $[\alpha / \mathrm{Fe}]$ vs. $[\mathrm{Fe} / \mathrm{H}] \mathrm{di}-$ agram. Even with this small sample and the sizeable systematic and statistical uncertainties attached to our age estimates, we can place reliable constraints on the chemical evolution of the Milky Way stellar disc:

1. In accordance with previous work, we find strong signatures of inside-out formation of the Galactic disc.

2 . When we compared our results to a multi-zone chemicalevolution model that treats the thin and thick disc separately, 

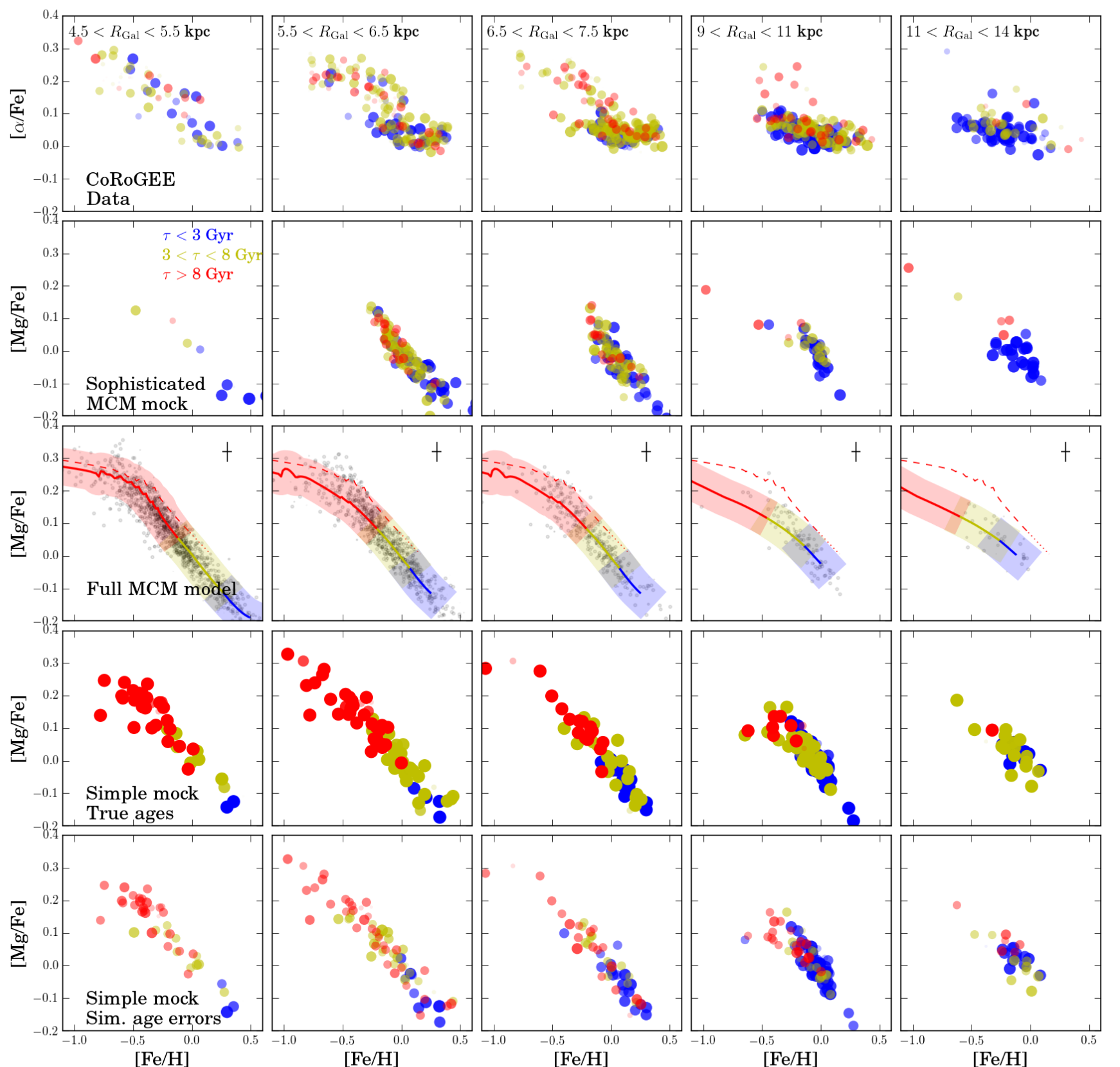

Fig. 17. $[\alpha / \mathrm{Fe}]$-vs.-[Fe/H] diagram for five different bins in Galactocentric distance $R_{\mathrm{Gal}}$. Top row: the CoRoGEE sample. The colours represent our stellar age estimates: blue indicates stars younger than $3 \mathrm{Gyr}$, red stands for stars older than $8 \mathrm{Gyr}$, and yellow for intermediate ages. The point size and transparency of each data point encode the age uncertainty, i.e., a smaller and more transparent symbol corresponds to a smaller probability of belonging to the particular age bin. Second row: the sophisticated MCM mock sample. Third row: all mock particles from the MCM $N$-body simulation, shown as grey dots. The solid lines represent the predictions of the underlying thin disc chemical-evolution model by Chiappini (2009) for stars born in the corresponding Galactocentric distance bin. The colour-code represents the age; the shaded regions along the lines correspond to a $2 \sigma$-confidence band, given the typical uncertainties in $[\mathrm{Fe} / \mathrm{H}]$ and $[\alpha / \mathrm{Fe}]$. The dashed lines show the chemical tracks of Chiappini (2009) for the thick disc. The error bar in the upper right of each panel represents the typical (internal) uncertainty of the chemical abundances. Fourth row: the simple MCM mock sample, without simulated age uncertainties. Last row: the simple MCM mock sample, with age uncertainties.

we found that the thin-disc models generally provide a good description of the main abundance-age trends, with the exception of the flat $[\alpha / \mathrm{Fe}]$ trend at high metallicity. The results improved when the stellar guiding-centre radius was used instead of the current Galactocentric distance.

3. In agreement with previous studies, we find that these pure chemical-evolution models fail to reproduce several important features seen in the data, such as the existence of SMR stars $([\mathrm{Fe} / \mathrm{H}]>0.2)$ in the solar neighbourhood and beyond, the exact shape of the $[\alpha / \mathrm{Fe}]-[\mathrm{Fe} / \mathrm{H}]$ distribution (in particular in the inner regions of the disc), and the existence of $[\alpha / \mathrm{Fe}]$-rich young stars.

4. When we compared our results with the predictions of the chemo-dynamical model of Minchev et al. (2013, 2014b), we found that the radial mixing in the model is not efficient enough to account for the number of SMR stars in the outer disc. Either a stronger radial mixing or the inclusion of a thick disc/bulge that formed stars for more than $3 \mathrm{Gyr}$ and 
produced SMR stars may resolve this discrepancy. In addition, the age distribution of the $[\alpha / \mathrm{Fe}]$-enhanced sequence in the CoRoGEE inner-disc field is much broader than expected from a combination of radial mixing and observational errors. Evolved blue stragglers may account for part of this population (Jofré et al. 2016; Yong et al. 2016), but do not offer an explanation for the different number counts in the inner and outer disc (Chiappini et al. 2015). Again, a thickdisc/bulge component with a more complex star-formation history than predicted by standard models might explain this observation.

In summary, we have demonstrated that the CoRoGEE sample is well-suited for the purpose of reconstructing the chemical enrichment history of the Milky Way disc. This first study will be followed by an investigation that focusses on exploring the detailed multi-element abundance patterns provided by APOGEE. It will be based on an analysis of newly reduced CoRoT light curves, resulting in more accurate seismic parameters, and will also include data from the CoRoT long run in the LRa02 field.

From the mid-term perspective, the CoRoGEE dataset can be viewed as a pathfinder and complementary dataset to the massive surveys that the Kepler-2 mission (K2; Howell et al. 2014) is currently conducting. The K2 Galactic Archaeology Program (Stello et al. 2015) will deliver seismic parameters for thousands of red giants in ten fields along the ecliptic plane, and, combined with the legacy of CoRoT as well as the original Kepler mission, will enable further improvements in the coverage of the Galactic disc with solar-like oscillating red giants.

Acknowledgements. F.A. would like to dedicate this work to the memory of Prof Angelo Cassatella ${ }^{\dagger}$. He also thanks E. C. Herenz for stimulating discussions and critical thoughts that accompanied him during the past months. TSR acknowledges support from CNPq-Brazil. B.M., F.B., R.P. and R.A.G. acknowledge financial support from the ANR program IDEE Interaction Des Étoiles et des Exoplanètes. J.M. acknowledges support from the ERC Consolidator Grant funding scheme (project STARKEY, G.A. No. 615604). L.G. and T.S.R. acknowledge partial support from PRIN INAF 2014 - CRA 1.05.01.94.05. T.M. acknowledges financial support from Belspo for contract PRODEX Gaia-DPAC. A.E.G.P., C.A.P., D.A.G.H., and O.Z. acknowledge support provided by the Spanish Ministry of Economy and Competitiveness (MINECO) under grants AYA2014 56359-P, RYC-2013-14182, and AYA-2014-58082-P. T.C.B. acknowledges partial support from grant PHY 14-30152 (Physics Frontier Center/JINA-CEE) awarded from the US National Science Foundation. S.a.M. acknowledges support from the NASA grant NNX12AE17G. S.z.M. has been supported by the János Bolyai Research Scholarship of the Hungarian Academy of Sciences. The research leading to the presented results has received funding from the European Research Council under the European Community's Seventh Framework Programme (FP7/2007-2013)/ERC grant agreement No. 338251 (StellarAges). The CoRoT space mission, launched on December 27 2006, was developed and operated by CNES, with the contribution of Austria, Belgium, Brazil, ESA (RSSD and Science Program), Germany and Spain. This research has made use of the ExoDat Database, operated at LAM-OAMP, Marseille, France, on behalf of the CoRoT/Exoplanet program. Funding for the SDSS-III Brazilian Participation Group has been provided by the Ministério de Ciência e Tecnologia (MCT), Fundação Carlos Chagas Filho de Amparo à Pesquisa do Estado do Rio de Janeiro (FAPERJ), Conselho Nacional de Desenvolvimento Científico e Tecnológico $(\mathrm{CNPq})$, and Financiadora de Estudos e Projetos (FINEP). Funding for SDSS-III has been provided by the Alfred P. Sloan Foundation, the Participating Institutions, the National Science Foundation, and the U.S. Department of Energy Office of Science. The SDSS-III web site is http://www.sdss3.org/. SDSS III is managed by the Astrophysical Research Consortium for the Participating Institutions of the SDSS-III Collaboration including the University of Arizona, the Brazilian Participation Group, Brookhaven National Laboratory, Carnegie Mellon University, University of Florida, the French Participation Group, the German Participation Group, Harvard University, the Instituto de Astrofisica de Canarias, the Michigan State/Notre Dame/JINA Participation Group, Johns Hopkins University, Lawrence Berkeley National Laboratory, Max Planck Institute for Astrophysics, Max Planck Institute for Extraterrestrial Physics, New Mexico State University, New York University, Ohio State University, Pennsylvania State University, University of Portsmouth, Princeton University, the Spanish Participation Group, University of Tokyo, University of Utah, Vanderbilt University, University of Virginia, University of Washington, and Yale University.

\section{References}

Abadi, M. G., Navarro, J. F., Steinmetz, M., \& Eke, V. R. 2003, ApJ, 597, 21 Alam, S., Albareti, F. D., Allende Prieto, C., Anders, F., \& Anderson, S. F., et al. 2015, ApJS, 219, 12

Allende Prieto, C., \& Lambert, D. L. 1999, A\&A, 352, 555

Anders, F., Chiappini, C., Santiago, B. X., et al. 2014, A\&A, 564, A115

Anders, F., Chiappini, C., Minchev, I., et al. 2016a, A\&A, submitted [arXiv: 1608.04951]

Anders, F., Chiappini, C., Rodrigues, T. S., et al. 2016b, Astron. Nachr., 337, 926 Baglin, A., Auvergne, M., Barge, P., et al. 2006, in ESA SP, eds. M. Fridlund, A. Baglin, J. Lochard, \& L. Conroy, 1306, 33

Barbuy, B., \& Grenon, M. 1990, in ESO Conf. and Workshop Proc., 35, eds. B. J. Jarvis, \& D. M. Terndrup, 83

Batalha, N. M., Borucki, W. J., Bryson, S. T., et al. 2011, ApJ, 729, 27

Bedding, T. R., Mosser, B., Huber, D., et al. 2011, Nature, 471, 608

Belkacem, K., Goupil, M. J., Dupret, M. A., et al. 2011, A\&A, 530, A142

Belkacem, K., Samadi, R., Mosser, B., Goupil, M.-J., \& Ludwig, H.-G. 2013 , in Progress in Physics of the Sun and Stars: A New Era in Helio- and Asteroseismology, eds. H. Shibahashi, \& A. E. Lynas-Gray, ASP Conf. Ser., 479,61

Bensby, T., Feltzing, S., \& Lundström, I. 2003, A\&A, 410, 527

Bensby, T., Alves-Brito, A., Oey, M. S., Yong, D., \& Meléndez, J. 2011, ApJ, 735, L46

Binney, J., \& Sanders, J. L. 2016, Astron. Nachr., 337, 939

Bovy, J., Rix, H.-W., Liu, C., et al. 2012, ApJ, 753, 148

Bovy, J., Nidever, D. L., Rix, H.-W., et al. 2014, ApJ, 790, 127

Bressan, A., Marigo, P., Girardi, L., et al. 2012, MNRAS, 427, 127

Brook, C. B., Stinson, G. S., Gibson, B. K., et al. 2012, MNRAS, 426, 690

Broomhall, A.-M., Miglio, A., Montalbán, J., et al. 2014, MNRAS, 440, 1828

Brown, T. M., Gilliland, R. L., Noyes, R. W., \& Ramsey, L. W. 1991, ApJ, 368, 599

Brunetti, M., Chiappini, C., \& Pfenniger, D. 2011, A\&A, 534, A75

Cardelli, J. A., Clayton, G. C., \& Mathis, J. S. 1989, ApJ, 345, 245

Carraro, G., Ng, Y. K., \& Portinari, L. 1998, MNRAS, 296, 1045

Casagrande, L., Schönrich, R., Asplund, M., et al. 2011, A\&A, 530, A138

Casagrande, L., Silva Aguirre, V., Stello, D., et al. 2014, ApJ, 787, 110

Casagrande, L., Silva Aguirre, V., Schlesinger, K. J., et al. 2016, MNRAS, 455, 987

Catelan, M. 2009, in IAU Symp. 258, eds. E. E. Mamajek, D. R. Soderblom, \& R. F. G. Wyse, 209

Chaplin, W. J., \& Miglio, A. 2013, ARA\&A, 51, 353

Chaplin, W. J., Kjeldsen, H., Christensen-Dalsgaard, J., et al. 2011, Science, 332, 213

Chen, L., Hou, J. L., \& Wang, J. J. 2003, AJ, 125, 1397

Cheng, J. Y., Rockosi, C. M., Morrison, H. L., et al. 2012, ApJ, 752, 51

Chiappini, C. 2009, in IAU Symp. 254, eds. J. Andersen, B. Nordström, \&

J. Bland-Hawthorn, 191

Chiappini, C., Matteucci, F., \& Gratton, R. 1997, ApJ, 477, 765

Chiappini, C., Matteucci, F., \& Romano, D. 2001, ApJ, 554, 1044

Chiappini, C., Anders, F., Rodrigues, T. S., et al. 2015, A\&A, 576, L12

Christensen-Dalsgaard, J. 1988, in Advances in Helio- and Asteroseismology, eds. J. Christensen-Dalsgaard, \& S. Frandsen, IAU Symp., 123, 295

Christensen-Dalsgaard, J. 1993, in GONG 1992. Seismic Investigation of the Sun and Stars, ed. T. M. Brown, ASP Conf. Ser., 42, 347

Cutri, R. M., Skrutskie, M. F., van Dyk, S., et al. 2003, 2MASS All Sky Catalog of point sources (NASA/IPAC)

Cutri, R. M., Wright, E. L., Conrow, T., et al. 2013, Explanatory Supplement to the AllWISE Data Release Products, Tech. Rep.

da Silva, L., Girardi, L., Pasquini, L., et al. 2006, A\&A, 458, 609

Damiani, C., Meunier, J.-C., Moutou, C., et al. 2016, A\&A, 595, A95

Davenport, J. R. A., Ivezić, Ž., Becker, A. C., et al. 2014, MNRAS, 440, 3430

Deheuvels, S. 2015, in 18th Cambridge Workshop on Cool Stars, Stellar Systems, and the Sun, 18, eds. G. T. van Belle, \& H. C. Harris, 489

Edvardsson, B., Andersen, J., Gustafsson, B., et al. 1993, A\&A, 275, 101

Eisenstein, D. J., Weinberg, D. H., Agol, E., et al. 2011, AJ, 142, 72

Freeman, K., \& Bland-Hawthorn, J. 2002, ARA\&A, 40, 487

Fuhrmann, K. 1998, A\&A, 338, 161

Gilmore, G. 1999, Balt. Astron., 8, 203

Gilmore, G. 2012, in Galactic Archaeology: Near-Field Cosmology and the Formation of the Milky Way, eds. W. Aoki, M. Ishigaki, T. Suda, T. Tsujimoto, \& N. Arimoto, ASP Conf. Ser., 458, 147

Girardi, L., Dalcanton, J., Williams, B., et al. 2008, PASP, 120, 583

Gough, D. O. 2001, in Astrophysical Ages and Times Scales, eds. T. von Hippel, C. Simpson, \& N. Manset, ASP Conf. Ser., 245, 31

Gratton, R., Carretta, E., Matteucci, F., \& Sneden, C. 1996, in Formation of the Galactic Halo...Inside and Out, eds. H. L. Morrison \& A. Sarajedini, ASP Conf. Ser., 92, 307 
Green, G. M., Schlafly, E. F., Finkbeiner, D. P., et al. 2015, ApJ, 810, 25 Grenon, M. 1972, in Proc. IAU Colloq. 17 on Âge des Étoiles, eds. G. Cayrel de Strobel, \& A. M. Delplace, 55

Grenon, M. 1989, Ap\&SS, 156, 29

Grenon, M. 1999, Ap\&SS, 265, 331

Guedes, J., Callegari, S., Madau, P., \& Mayer, L. 2011, ApJ, 742, 76

Gunn, J. E., Siegmund, W. A., Mannery, E. J., et al. 2006, AJ, 131, 2332

Hayden, M. R., Holtzman, J. A., Bovy, J., et al. 2014, AJ, 147, 116

Hayden, M. R., Bovy, J., Holtzman, J. A., et al. 2015, ApJ, 808, 132

Hekker, S., Kallinger, T., Baudin, F., et al. 2009, A\&A, 506, 465

Henden, A., \& Munari, U. 2014, Contributions of the Astronomical Observatory Skalnate Pleso, 43, 518

Hill, V., Plez, B., Cayrel, R., et al. 2002, A\&A, 387, 560

Holmberg, J., Nordström, B., \& Andersen, J. 2007, A\&A, 475, 519

Holtzman, J. A., Shetrone, M., Johnson, J. A., et al. 2015, AJ, 150, 148

Hou, J. L., Prantzos, N., \& Boissier, S. 2000, A\&A, 362, 921

Howell, S. B., Sobeck, C., Haas, M., et al. 2014, PASP, 126, 398

Ivezić, Ż., Connolly, A., VanderPlas, J., \& Gray, A. 2013, Statistics, Data Mining, and Machine Learning in Astronomy

Jofré, P., Jorissen, A., Van Eck, S., et al. 2016, A\&A, 595, A60

Jørgensen, B. R., \& Lindegren, L. 2005, A\&A, 436, 127

Kaiser, N., Burgett, W., Chambers, K., et al. 2010, in SPIE Conf. Ser., 733

Kjeldsen, H., \& Bedding, T. R. 1995, A\&A, 293, 87

Kordopatis, G., Binney, J., Gilmore, G., et al. 2015, MNRAS, 447, 3526

Kotoneva, E., Flynn, C., Chiappini, C., \& Matteucci, F. 2002, MNRAS, 336, 879

Kubryk, M., Prantzos, N., \& Athanassoula, E. 2015a, A\&A, 580, A126

Kubryk, M., Prantzos, N., \& Athanassoula, E. 2015b, A\&A, 580, A127

Lebreton, Y., \& Goupil, M. J. 2014, A\&A, 569, A21

Lebreton, Y., Goupil, M. J., \& Montalbán, J. 2014, in EAS Pub. Ser., 65, 99

Majewski, S. R., Zasowski, G., \& Nidever, D. L. 2011, ApJ, 739, 25

Majewski, S. R., Schiavon, R. P., Frinchaboy, P. M., et al. 2016, Astron. Nachr. 337, 863

Mannucci, F., Della Valle, M., \& Panagia, N. 2006, MNRAS, 370, 773

Marigo, P., Girardi, L., Bressan, A., et al. 2008, A\&A, 482, 883

Martig, M., Rix, H.-W., Aguirre, V. S., et al. 2015, MNRAS, 451, 2230

Mathur, S., Metcalfe, T. S., Woitaszek, M., et al. 2012, ApJ, 749, 152

Matteucci, F. 2001, The chemical evolution of the Galaxy, Astrophysics Space Sci. Lib., 253

Matteucci, F., \& Romano, D. 1999, Ap\&SS, 265, 311

Metcalfe, T. S., Monteiro, M. J. P. F. G., Thompson, M. J., et al. 2010, ApJ, 723, 1583

Metcalfe, T. S., Creevey, O. L., Doğan, G., et al. 2014, ApJS, 214, 27

Meunier, J.-C., Deleuil, M., Moutou, C., et al. 2007, in Astronomical Data Analysis Software and Systems XVI, eds. R. A. Shaw, F. Hill, \& D. J. Bell, ASP Conf. Ser., 376, 339

Michel, E., Baglin, A., Weiss, W. W., et al. 2008, Comm. Asteroseismol., 156, 73

Miglio, A. 2012, in Asteroseismology of Red Giants as a Tool for Studying Stellar Populations: First Steps, eds. A. Miglio, J. Montalbán, \& A. Noels (Berlin, Heidelberg: Springer Verlag), 11

Miglio, A., Montalbán, J., Baudin, F., et al. 2009, A\&A, 503, L21

Miglio, A., Chiappini, C., Morel, T., et al. 2013a, in Eur. Phys. J. Web Conf., 43, 3004

Miglio, A., Chiappini, C., Morel, T., et al. 2013b, MNRAS, 429, 423

Miglio, A., Eggenberger, P., Girardi, L., \& Montalbán, J. 2015, Astrophys. Space Science Proc. (Springer), 39

Miglio, A., Chaplin, W. J., Brogaard, K., et al. 2016, MNRAS, 461, 760

Minchev, I., Chiappini, C., \& Martig, M. 2013, A\&A, 558, A9

Minchev, I., Chiappini, C., \& Martig, M. 2014a, A\&A, 572, A92

Minchev, I., Chiappini, C., \& Martig, M. 2014b, in IAU Symp. 298, eds. S. Feltzing, G. Zhao, N. A. Walton, \& P. Whitelock, 130

Monet, D. G., Levine, S. E., Canzian, B., et al. 2003, AJ, 125, 984

Montalbán, J., \& Noels, A. 2013, in Eur. Phys. J. Web Conf., 43, 3002

Montalbán, J., Miglio, A., Noels, A., et al. 2013, ApJ, 766, 118

Mosser, B., \& Appourchaux, T. 2009, A\&A, 508, 877

Mosser, B., Belkacem, K., Goupil, M.-J., et al. 2010, A\&A, 517, A22

Mosser, B., Barban, C., Montalbán, J., et al. 2011, A\&A, 532, A86

Ng, Y. K., \& Bertelli, G. 1998, A\&A, 329, 943

Nidever, D. L., Bovy, J., Bird, J. C., et al. 2014, ApJ, 796, 38

Nidever, D. L., Holtzman, J. A., Allende Prieto, C., et al. 2015, AJ, 150, 173

Nishiyama, S., Tamura, M., Hatano, H., et al. 2009, ApJ, 696, 1407

Noels, A., \& Bragaglia, A. 2015, Astrophys. Space Sci. Proc., 39, 167

Nordström, B., Mayor, M., Andersen, J., et al. 2004, A\&A, 418, 989

O’Donnell, J. E. 1994, ApJ, 422, 158

Pagel, B. E. J. 1997, Nucleosynthesis and Chemical Evolution of Galaxies (Cambridge University Press)

Peek, J. E. G., \& Graves, G. J. 2010, ApJ, 719, 415
Perryman, M. A. C., de Boer, K. S., Gilmore, G., et al. 2001, A\&A, 369, 339 Piffl, T. 2013, Ph.D. Thesis, Universität Potsdam

Pinsonneault, M. H., Elsworth, Y., Epstein, C., et al. 2014, ApJS, 215, 19 Ramírez, I., Allende Prieto, C., \& Lambert, D. L. 2007, A\&A, 465, 271

Rauer, H., Catala, C., Aerts, C., et al. 2014, Exp. Astron., 38, 249

Reimers, D. 1975, Mem. Soc. Roy. Sci. Liege, 8, 369

Roca-Fàbrega, S., Valenzuela, O., Colín, P., et al. 2016, ApJ, 824, 94

Rocha-Pinto, H. J., \& Maciel, W. J. 1996, MNRAS, 279, 447

Rodrigues, T. S., Girardi, L., Miglio, A., et al. 2014, MNRAS, 445, 2758

Salaris, M., Chieffi, A., \& Straniero, O. 1993, ApJ, 414, 580

Santiago, B. X., Brauer, D. E., Anders, F., et al. 2016, A\&A, 585, A42

Scannapieco, C., Wadepuhl, M., Parry, O. H., et al. 2012, MNRAS, 423, 1726

Scannapieco, C., Creasey, P., Nuza, S. E., et al. 2015, A\&A, 577, A3

Schlafly, E. F., \& Finkbeiner, D. P. 2011, ApJ, 737, 103

Schlafly, E. F., Green, G., Finkbeiner, D. P., et al. 2014, ApJ, 789, 15

Schlegel, D. J., Finkbeiner, D. P., \& Davis, M. 1998, ApJ, 500, 525

Schönrich, R., \& Binney, J. 2009, MNRAS, 396, 203

Schultheis, M., Zasowski, G., Allende Prieto, C., et al. 2014, AJ, 148, 24

Sharma, S., Bland-Hawthorn, J., Johnston, K. V., \& Binney, J. 2011, ApJ, 730, 3

Silva Aguirre, V., Basu, S., Brandão, I. M., et al. 2013, ApJ, 769, 141

Soderblom, D. 2013, in Asteroseismology of Stellar Populations in the Milky Way (Springer)

Soderblom, D. R. 2010, ARA\&A, 48, 581

Soubiran, C. 1999, Ap\&SS, 265, 353

Stasińska, G., Prantzos, N., Meynet, G., et al. 2012, Oxygen in the Universe, EAS Publ. Ser., 54

Steinmetz, M., Zwitter, T., Siebert, A., et al. 2006, AJ, 132, 1645

Stello, D., Huber, D., Sharma, S., et al. 2015, ApJ, 809, L3

Stinson, G. S., Bailin, J., Couchman, H., et al. 2010, MNRAS, 408, 812

Takeda, G., Ford, E. B., Sills, A., et al. 2007, ApJS, 168, 297

Tassoul, M. 1980, ApJS, 43, 469

Trevisan, M., Barbuy, B., Eriksson, K., et al. 2011, A\&A, 535, A42

Turon, C., Primas, F., Binney, J., et al. 2008, ESA-ESO Working Group on Galactic Populations, Chemistry and Dynamics, Tech. Rep.

Twarog, B. A. 1980, ApJ, 242, 242

Ulrich, R. K. 1986, ApJ, 306, L37

White, T. R., Bedding, T. R., Stello, D., et al. 2011, ApJ, 743, 161

Wilson, J. C., Hearty, F., Skrutskie, M. F., et al. 2010, in SPIE Conf. Ser., 7735

Wilson, J. C., Hearty, F., Skrutskie, M. F., et al. 2012, in SPIE Conf. Ser., 8446

Yong, D., Casagrande, L., Venn, K. A., et al. 2016, MNRAS, 459, 487

Zacharias, N., Finch, C. T., Girard, T. M., et al. 2013, AJ, 145, 44

Zamora, O., García-Hernández, D. A., Allende Prieto, C., et al. 2015, AJ, 149, 181

Zasowski, G., Johnson, J. A., Frinchaboy, P. M., et al. 2013, AJ, 146, 81

Zhao, G., Zhao, Y.-H., Chu, Y.-Q., Jing, Y.-P., \& Deng, L.-C. 2012, RA\&A, 12, 723

Zucker, D. B., de Silva, G., Freeman, K., Bland-Hawthorn, J., \& Hermes Team. 2012, in Galactic Archaeology: Near-Field Cosmology and the Formation of the Milky Way, eds. W. Aoki, M. Ishigaki, T. Suda, T. Tsujimoto, \& N. Arimoto, ASP Conf. Ser., 458, 421

1 Leibniz-Institut für Astrophysik Potsdam (AIP), An der Sternwarte 16, 14482 Potsdam, Germany e-mail: [fanders; cristina.chiappini]@aip.de

2 Laboratório Interinstitucional de e-Astronomia, - LIneA, Rua Gal. José Cristino 77, 20921-400 Rio de Janeiro, Brazil

3 Osservatorio Astronomico di Padova - INAF, Vicolo dell'Osservatorio 5, 35122 Padova, Italy e-mail: thaise.rodrigues@oapd.inaf.it

4 Dipartimento di Fisica e Astronomia, Università di Padova, Vicolo dell'Osservatorio 2, 35122 Padova, Italy

5 School of Physics and Astronomy, University of Birmingham, Edgbaston, Birmingham, B15 2TT, UK

6 LESIA, Observatoire de Paris, PSL Research University, CNRS, Université Pierre et Marie Curie, Université Denis Diderot, 92195 Meudon, France

7 Institut d'Astrophysique et de Géophysique, Allée du 6 aout, 17 Bat. B5c, 4000 Liège 1 (Sart-Tilman), Belgium

8 The Ohio State University, Department of Astronomy, 4055 McPherson Laboratory, 140 West 18th Ave., Columbus, OH 432101173, USA

9 Observatoire de la Côte d'Azur, Laboratoire Lagrange, CNRS UMR 7923, BP 4229, 06304 Nice Cedex, France 
${ }^{10}$ Institut d'Astrophysique Spatiale, CNRS, Université Paris XI, 91405 Orsay Cedex, France

11 Max-Planck-Institut für Sonnensystemforschung, Justus-vonLiebig-Weg 3, 37077 Göttingen, Germany

12 Stellar Astrophysics Centre, Department of Physics and Astronomy, Aarhus University, Ny Munkegade 120, 8000 Aarhus C, Denmark

13 Institut für Astronomie, Universität Wien, Türkenschanzstr. 17, Wien, Austria

${ }^{14}$ Laboratoire AIM, CEA/DRF - CNRS - Univ. Paris Diderot IRFU/SAp, Centre de Saclay, 91191 Gif-sur-Yvette Cedex, France

15 Space Science Institute, 4750 Walnut Street Suite 205, Boulder CO 80301, USA

16 Instituto de Física, Universidade Federal do Rio Grande do Sul, Caixa Postal 15051, 91501-970 Porto Alegre, Brazil

17 Max-Planck-Institut für Astronomie, Königstuhl 17, 69117 Heidelberg, Germany

18 Observatório Nacional, Rua Gal. José Cristino 77, 20921-400 Rio de Janeiro, Brazil

19 Instituto de Astrofisica de Canarias, C/ Vía Láctea, s/n, 38205 La Laguna, Tenerife, Spain

20 Departamento de Astrofísica, Universidad de La Laguna (ULL), 38206 La Laguna, Tenerife, Spain

21 Dept. of Physics and JINA-CEE: Joint Institute for Nuclear Astrophysics - Center for the Evolution of the Elements, Univ. of Notre Dame, Notre Dame, IN 46530, USA
22 Department of Astronomy, Case Western Reserve University, Cleveland, OH 44106, USA

23 New Mexico State University, Las Cruces, NM 88003, USA

24 Department of Astronomy, University of Virginia, PO Box 400325, Charlottesville VA 22904-4325, USA

25 ELTE Gothard Astrophysical Observatory, Szent Imre herceg st. 112, 9704 Szombathely, Hungary

26 Department of Astronomy, Indiana University, Bloomington, IN 47405, USA

27 Dept. of Astronomy, University of Michigan, Ann Arbor, MI, 48104, USA

28 Apache Point Observatory, PO Box 59, Sunspot, NM 88349, USA

29 Astrophysics Research Institute, Liverpool John Moores University, IC2, Liverpool Science Park 146 Brownlow Hill Liverpool L3 5RF, UK

30 Department of Astronomy and Astrophysics, The Pennsylvania State University, University Park, PA 16802, USA

31 Institute for Gravitation and the Cosmos, The Pennsylvania State University, University Park, PA 16802, USA

32 Mcdonald Observatory, University of Texas at Austin, HC75 Box 1337-MCD, Fort Davis, TX 79734, USA

33 Vanderbilt University, Dept. of Physics \& Astronomy, VU Station B 1807, Nashville, TN 37235, USA

34 Johns Hopkins University, Dept. of Physics and Astronomy, 3701 San Martin Drive, Baltimore, MD 21210, USA 


\section{Appendix A: PARAM sanity checks}

\section{A.1. Seismic vs. spectroscopic gravities}

Figure 4 shows a comparison between seismic and (calibrated) ASPCAP $\log g$ as a function of effective temperature. An immediate result is that while asteroseismology provides an accurate benchmark for spectroscopic gravities, spectroscopy may serve as an important cross-check for the determined asteroseismic parameters, especially for fainter stars. By requiring that the difference in $\log g$ not be too large, we are able to sort out potentially flawed seismic (or spectroscopic) parameters. For DR12, the ASPCAP gravities were calibrated using seismic gravities from Kepler (Holtzman et al. 2015). An analysis of APOKASC stars with known evolutionary status demonstrated that for RGB stars that have not yet entered the helium-burning phase, the offset between seismic and spectroscopic gravity is larger than for red-clump (RC) stars. Hence, one would ideally use two different calibration relations for the RC and RGB stars. In the meantime, ASPCAP provides a $\log g$ calibration only for RGB stars, while a calibration for RC stars is reported in a separate catalogue (Bovy et al. 2014). The temperature dependence of the gravity offset also reflects the bias imposed by the adopted calibration relation: at lower temperatures (on the upper RGB), the systematic discrepancy vanishes.

\section{A.2. Scaling relations vs. grid-based results}

The concordance between the results obtained with PARAM and from the direct method has already been mentioned in Rodrigues et al. (2014), who used PARAM to estimate masses, radii, and gravities for the APOKASC sample. In the direct method, the quantities mass, radius, and gravity are calculated through seismic scaling relations (which involve seismic global parameters, and $T_{\text {eff }}$, but no information on metallicity or stellar models).

Figure A.1 presents the comparison of the two methods for our sample. The resulting mean differences and rms scatter are $(5.3 \pm 13.7) \%$ in mass, $(1.3 \pm 5.1) \%$ in radius, and $0.005 \pm 0.012 \mathrm{dex}[0.2 \pm 0.5) \%]$ in $\log g$, comparable to what was reported by Rodrigues et al. (2014) for APOKASC.

\section{A.3. Comparison with extinction maps}

Another check is provided by Figs. A.2 and A.3 which show $A_{V}$ extinction maps for the sample stars in the two CoRoT fields, and compare these results to the maps obtained using other methods: The Rayleigh-Jeans colour excess (RJCE) method (Majewski et al. 2011; Zasowski et al. 2013), the isochronematching method presented in Schultheis et al. (2014), and the 2D dust extinction maps derived from Pan-STARRS1 photometry (Schlafly et al. 2014). A quantitative comparison between our results and these literature methods, together with empirical fitting formulae for each extinction scale, is presented in Fig. A.4. In summary, we can say the following:

- The RJCE method (Majewski et al. 2011) relies on the fact that the intrinsic NIR - mid-IR colours (e.g. $H_{2 M A S S}-$ $W 2_{\text {WISE }}$ ) of a star depend very little on the spectral type, and therefore the observed minus intrinsic colour provides a measurement of the amount of dust in the sightline of an observer. The comparison with the extinction values calculated using this recipe (which was used for APOGEE targeting; Zasowski et al. 2013) shows that - assuming a particular extinction law (Nishiyama et al. 2009) - RJCE overpredicts the amount of $V$-band extinction in both LRa01 and LRc01 by about $0.5 \mathrm{mag}$. Of course, as APOGEE operates in the $H$ band $\left(A_{H} / A_{V} \approx 1 / 6\right)$, this systematic difference is of minor importance for APOGEE targeting purposes. However, our comparison shows that, when computing distances to APOGEE field stars (e.g. Anders et al. 2014; Santiago et al. 2016), we should be cautious in using the targeting extinction values; in particular, distant low-latitude stars will be assigned systematically greater distances.

- The isochrone-based method of Schultheis et al. (2014), tailored to quantifying 3D extinction towards the Galactic bulge, yields slightly lower extinction values than our method; there is only a minor zero-point offset of about $0.05 \mathrm{mag}$ in the extinction scale with respect to PARAM (middle panel of Fig. A.4). When this effect is calibrated out, the rms scatter around the mean relation is about 0.2 mag in both fields.

- Schlafly et al. (2014) used multi-band photometry starcounts from Pan-STARRS1 (Kaiser et al. 2010) to create a 2D $E(B-V)$ reddening map, quantifying integrated interstellar extinction at heliocentric distances of $4.5 \mathrm{kpc}$. The resolution at low Galactic latitudes is typically $7^{\prime}$ and the systematic uncertainty in $E(B-V)$ around $0.03 \mathrm{mag}$. Our results show that while the overall amount of extinction for the bulk of the CoRoGEE sample is reproduced by the PanSTARRS maps, the relation between our extinction estimates and those derived from Pan-STARRS is dominated by considerable scatter, especially in the LRc01 field. This result is expected, as most of our stars lie within the $4.5 \mathrm{kpc}$ boundary, some even closer than $1 \mathrm{kpc}$ from the Sun.

- Not shown in Fig. A.4 is the comparison of our results with the classical 2D extinction SFD maps of Schlegel, Finkbeiner, \& Davis (1998), as for Galactic astronomy purposes, they are surpassed in accuracy by the maps of Schlafly et al. (2014). It is worth mentioning, however, that in the LRc01 field (only $7^{\circ}$ off the Galactic plane) our method agrees well with the SFD maps, also on a star-by-star level; we find a very tight relation between $A_{V, \mathrm{SFD}}$ and $A_{V, \text { PARAM }}$ in this field, with an rms scatter of $\sim 0.15 \mathrm{mag}$. This suggests that the extinction in this field is likely to be dominated by a nearby foreground cloud (as also visible in the WISE image of Fig. 1).

In the LRa01 field, however, the situation is not as favourable: The SFD maps overpredict the extinction in LRa01 by more than one magnitude on average, and the correlation with the PARAM results is marginal. This finding agrees with previous studies close to Galactic plane (e.g. Peek \& Graves 2010; Schlafly \& Finkbeiner 2011), and might be explained by significant additional amounts of dust beyond the bulk of the CoRoGEE stars (e.g. the Galactic warp). 
F. Anders et al.: Galactic archaeology with CoRoT and APOGEE
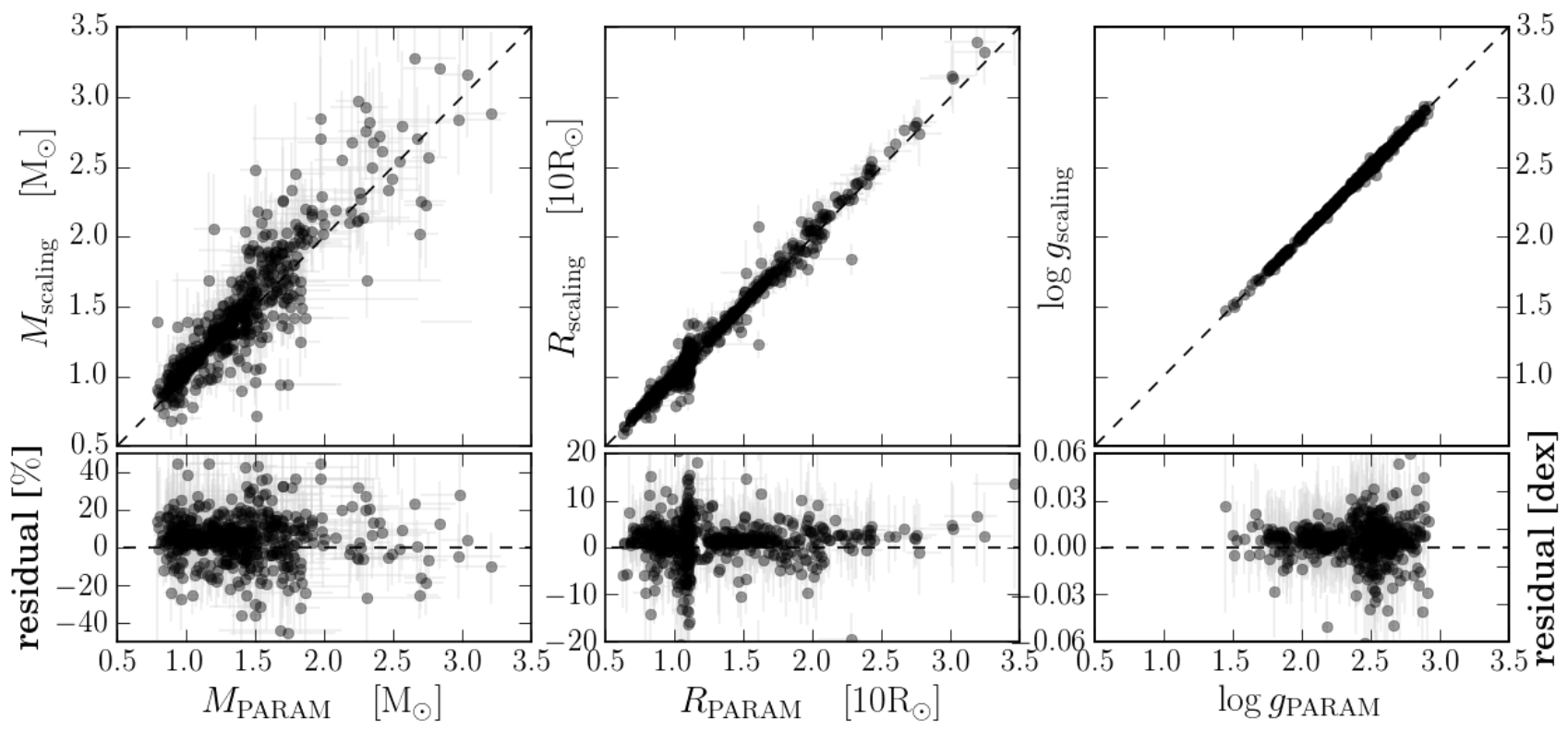

Fig. A.1. Comparison of our PARAM results for mass (left panel), radius (middle), and surface gravity (right) with the results obtained using seismic scaling relations. Compare also Fig. 4 of Rodrigues et al. (2014).

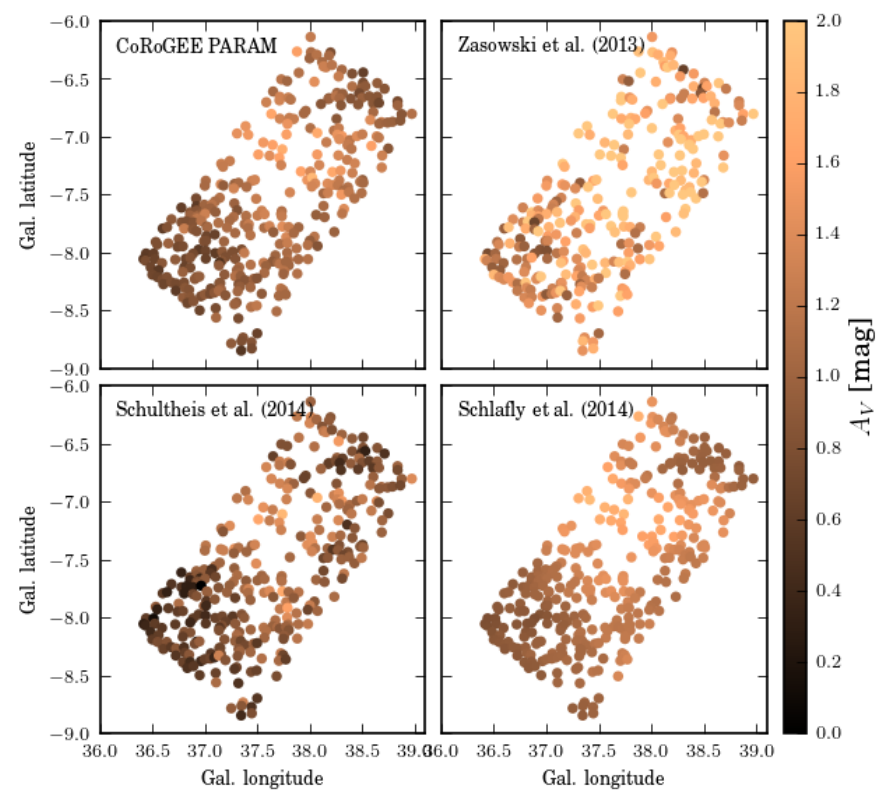

Fig. A.2. Comparison of our derived individual $A_{V}$ extinction values for stars in the LRc01 field with extinction estimates derived by other (mostly independent) methods.



Fig. A.3. Same as Fig. A.2, now for the LRa01 field. 



Fig. A.4. Comparison of our PARAM extinctions with the results obtained by the RJCE method (Zasowski et al. 2013; left panel), isochrone matching (Schultheis et al. 2014; middle panel), and the Pan-STARRS1 dust maps of Schlafly et al. (2014). As before, stars in LRa01 are plotted in blue, while LRc01 stars are plotted in red. The corresponding robust linear fits (using a Huber loss function; see e.g. Ivezić et al. 2013) are shown as solid lines, with the fit coefficients indicated in each panel. 


\section{Appendix B: Released data}

In Table B.1, we shortly summarise the contents of this first set of CoRoGEE data that is released through the CDS Vizier Catalogue Service ${ }^{19}$.

The present CoRoT-APOGEE dataset contains a large amount of information (206 columns) on the 606 successfully observed stars. In addition to the measurements derived directly from APOGEE and CoRoT observations, we include photometry from OBSCAT, APASS, SDSS, 2MASS, and WISE, information from the EXODAT archive, stellar parameters, distances and extinctions from PARAM and/or seismic scaling relations, cross-matches to the APOGEE DR12 RC catalogue (Bovy et al. 2014), the UCAC-4 catalogue (Zacharias et al. 2013), and additional information on the kinematics of the stars.

\footnotetext{
19 vizier.u-strasbg.fr/viz-bin/VizieR
} 
Table B.1. Description and explanation of the contents of the CoRoT-APOGEE catalogue.

\begin{tabular}{|c|c|c|c|}
\hline No. Column & Data type & ape Unit & Description \\
\hline \$1 CoRoT_ID & Integer & & CoRoT Star Identifier - 10 digits number \\
\hline$\$ 2$ APOGEE_ID & String & & APOGEE Identifier \\
\hline$\$ 3$ RA & Double & deg & Right Ascension (J2000.0, from 2MASS) \\
\hline$\$ 4 \mathrm{Dec}$ & Double & deg & Declination (J2000.0, from 2MASS) \\
\hline$\$ 5$ GLON & Double & $\operatorname{deg}$ & Galactic Longitude \\
\hline$\$ 6$ GLAT & Double & deg & Galactic Latitude \\
\hline$\$ 7$ FIELD & String & & APOGEE Field \\
\hline$\$ 8$ run_id & String & & Identifier of the CoRoT mission run - format AAIIA \\
\hline$\$ 9$ Seismic_results & String & & $\begin{array}{l}\text { Fitting method used to fit the frequency power spectrum } \\
\text { (automatic or supervised) }\end{array}$ \\
\hline$\$ 10$ numax & Float & $\mathrm{muHz}$ & Frequency of maximum oscillation power \\
\hline \$11e_numax & Float & $\mathrm{muHz}$ & Z Uncertainty of the frequency of maximum oscillation power \\
\hline \$12 Dnu & Float & $\mathrm{muHz}$ & Large frequency separation \\
\hline \$13 e_Dnu & Float & $\mathrm{muHz}$ & z Uncertainty of the large frequency separation \\
\hline$\$ 14$ evstat & String & & Evolutionary stage (RGB: red-giant branch, or RC: red clump) \\
\hline \$15 ASPCAP_ID & String & & Unique ASPCAP identifier \\
\hline \$16 FILE & String & & File name of visit-combined APOGEE spectrum \\
\hline \$17 TELESCOPE & String & & $\begin{array}{l}\text { String representation of of telescope used for observation } \\
\text { (currently APO } 1 \mathrm{~m} \text { or } 2.5 \mathrm{~m} \text { ) }\end{array}$ \\
\hline \$18LOCATION_ID & Short & & APOGEE Field Location ID \\
\hline \$19TARGFLAGS & String & & APOGEE Target flags ${ }^{20}$ \\
\hline \$20 NVISITS & Integer & & Number of visits into combined spectrum \\
\hline \$21 SNR & Float & & median $\mathrm{S} / \mathrm{N}$ per pixel in combined spectrum \\
\hline \$22 STARFLAGS & String & & APOGEE Star flags ${ }^{21}$ \\
\hline \$23 VHELIO_AVG & Float & & $\begin{array}{l}\text { APOGEE average radial velocity, weighted by } \mathrm{S} / \mathrm{N} \text {, using } \mathrm{RVs} \\
\text { determined from cross-correlation of ind. spectra with template spectrum }\end{array}$ \\
\hline \$24 VSCATTER & Float & & Scatter of individual visit RVs around average \\
\hline \$25 VERR_MED & Float & & Median of individual visit RV errors \\
\hline \$26 PARAM & float[] & 7 & $\begin{array}{l}\text { Empirically calibrated parameter array, using ASPCAP stellar parameters } \\
\text { fit }+ \text { calibrations, in the order: } T_{\text {eff }}, \log g, v_{\text {micro }},[\mathrm{M} / \mathrm{H}],[\mathrm{C} / \mathrm{M}],[\mathrm{N} / \mathrm{M}],[\alpha / \mathrm{M}]\end{array}$ \\
\hline \$27 FPARAM & float[] & 7 & Uncalibrated output parameter array from ASPCAP stellar parameters fit \\
\hline \$28 PARAM_COV & float[] & 49 & $\begin{array}{l}\text { Covariance of calibrated parameters, but with only diagonal elements } \\
\text { from external uncertainty estimation }\end{array}$ \\
\hline \$29FPARAM_COV & float[] & 49 & Covariance of fitted parameters from ASPCAP \\
\hline \$30 TEFF & Double & $\mathrm{K}$ & APOGEE $T_{\text {eff }}$ from ASPCAP analysis of combined spectrum \\
\hline \$31 TEFF_ERR & Double & $\mathrm{K}$ & APOGEE $T_{\text {eff }}$ uncertainty \\
\hline$\$ 32 \mathrm{LOGG}$ & Float & [dex $]$ & APOGEE $\log g$ from ASPCAP analysis of combined spectrum \\
\hline \$33LOGG_ERR & Float & dex & APOGEE $\log g$ uncertainty \\
\hline \$34 PARAM_M_H & Float & {$[\mathrm{dex}]$} & APOGEE $[\mathrm{M} / \mathrm{H}]$ from ASPCAP analysis of combined spectrum \\
\hline \$35 MH_Anders & Float & {$[\mathrm{dex}]$} & APOGEE $[\mathrm{M} / \mathrm{H}]$ calibrated as in this paper and Santiago et al. (2016) \\
\hline \$36PARAM_M_H_ERR & Float & $\operatorname{dex}$ & APOGEE $[\mathrm{M} / \mathrm{H}]$ uncertainty \\
\hline \$37PARAM_AL̄PHA_A_M & Float & {$[\mathrm{dex}]$} & APOGEE $[\alpha / \mathrm{M}]$ from ASPCAP analysis of combined spectrum \\
\hline \$38PARAM_ALPHA_M_ERR & Float & dex & APOGEE $[\alpha / \mathrm{M}]$ uncertainty \\
\hline \$39 ASPCAP_CHI2 & Float & & Reduced $\chi^{2}$ of ASPCAP Fit \\
\hline \$40 ASPCAPFLAGS & String & & Flags for ASPCAP analysis \\
\hline \$41 AL_H & Float & dex & {$[\mathrm{Al} / \mathrm{H}]$ from ASPCAP analysis of combined spectrum } \\
\hline \$42CA_H & Float & dex & {$[\mathrm{Ca} / \mathrm{H}]$ from ASPCAP analysis of combined spectrum } \\
\hline \$43C_H & Float & $\operatorname{dex}$ & {$[\mathrm{C} / \mathrm{H}]$ from ASPCAP analysis of combined spectrum } \\
\hline \$44FE_H & Float & $\operatorname{dex}$ & {$[\mathrm{Fe} / \mathrm{H}]$ from ASPCAP analysis of combined spectrum } \\
\hline$\$ 45 \mathrm{~K} \_\mathrm{H}$ & Float & dex & {$[\mathrm{K} / \mathrm{H}]$ from ASPCAP analysis of combined spectrum } \\
\hline \$46MG_H & Float & dex & {$[\mathrm{Mg} / \mathrm{H}]$ from ASPCAP analysis of combined spectrum } \\
\hline \$47 MN_H & Float & $\operatorname{dex}$ & {$[\mathrm{Mn} / \mathrm{H}]$ from ASPCAP analysis of combined spectrum } \\
\hline \$48 NA_H & Float & $\operatorname{dex}$ & {$[\mathrm{Na} / \mathrm{H}]$ from ASPCAP analysis of combined spectrum } \\
\hline \$49 NI_H & Float & dex & {$[\mathrm{Ni} / \mathrm{H}]$ from ASPCAP analysis of combined spectrum } \\
\hline$\$ 50 \mathrm{~N} \_\mathrm{H}$ & Float & dex & {$[\mathrm{N} / \mathrm{H}]$ from ASPCAP analysis of combined spectrum } \\
\hline \$51O_H & Float & dex & {$[\mathrm{O} / \mathrm{H}]$ from ASPCAP analysis of combined spectrum } \\
\hline \$52SI_H & Float & dex & {$[\mathrm{Si} / \mathrm{H}]$ from ASPCAP analysis of combined spectrum } \\
\hline
\end{tabular}

Notes. ${ }^{(20)}$ https://wWW. sdss.org/dr12/algorithms/bitmasks/\#APOGEE_TARGET2 . ${ }^{(21)}$ https://wWW.sdss.org/dr12/algorithms/ bitmasks/\#APOGEE_STARFLAG 
Table B.1. continued.

\begin{tabular}{|c|c|c|c|}
\hline \multirow{2}{*}{$\begin{array}{l}\text { No. } \\
\$ 53\end{array}$} & \multirow{2}{*}{$\begin{array}{l}\text { Column } \\
\text { S_H }\end{array}$} & \multicolumn{2}{|c|}{ Data type Shape Unit Description } \\
\hline & & Float & dex $[\mathrm{S} / \mathrm{H}]$ from ASPCAP analysis of combined spectrum \\
\hline$\$ 54$ & TI_H & Float & $\operatorname{dex}[\mathrm{Ti} / \mathrm{H}]$ from ASPCAP analysis of combined spectrum \\
\hline$\$ 55$ & V_H & Float & $\operatorname{dex}[\mathrm{V} / \mathrm{H}]$ from ASPCAP analysis of combined spectrum \\
\hline$\$ 56$ & AL_H_ERR & Float & dex $[\mathrm{Al} / \mathrm{H}]$ uncertainty from ASPCAP analysis of combined spectrum \\
\hline$\$ 57$ & CA_H_ERR & Float & $\operatorname{dex}[\mathrm{Ca} / \mathrm{H}]$ uncertainty from ASPCAP analysis of combined spectrum \\
\hline$\$ 58$ & C_H_ERR & Float & dex $[\mathrm{C} / \mathrm{H}]$ uncertainty from ASPCAP analysis of combined spectrum \\
\hline$\$ 59$ & FE_H_ERR & Float & dex $[\mathrm{Fe} / \mathrm{H}]$ uncertainty from ASPCAP analysis of combined spectrum \\
\hline$\$ 60$ & K_H_ERR & Float & dex $[\mathrm{K} / \mathrm{H}]$ uncertainty from ASPCAP analysis of combined spectrum \\
\hline$\$ 61$ & MG_H_ERR & Float & dex $[\mathrm{Mg} / \mathrm{H}]$ uncertainty from ASPCAP analysis of combined spectrum \\
\hline$\$ 62$ & MN_H_ERR & Float & $\operatorname{dex}[\mathrm{Mn} / \mathrm{H}]$ uncertainty from ASPCAP analysis of combined spectrum \\
\hline$\$ 63$ & NA_H_ERR & Float & dex $[\mathrm{Na} / \mathrm{H}]$ uncertainty from ASPCAP analysis of combined spectrum \\
\hline$\$ 64$ & NI_H_ERR & Float & $\operatorname{dex}[\mathrm{Ni} / \mathrm{H}]$ uncertainty from ASPCAP analysis of combined spectrum \\
\hline$\$ 65$ & N_H_ERR & Float & dex $[\mathrm{N} / \mathrm{H}]$ uncertainty from ASPCAP analysis of combined spectrum \\
\hline$\$ 66$ & O_H_ERR & Float & $\operatorname{dex}[\mathrm{O} / \mathrm{H}]$ uncertainty from ASPCAP analysis of combined spectrum \\
\hline$\$ 67$ & SI_H_ERR & Float & $\operatorname{dex}[\mathrm{Si} / \mathrm{H}]$ uncertainty from ASPCAP analysis of combined spectrum \\
\hline$\$ 68$ & S_H_ERR & Float & dex $[\mathrm{S} / \mathrm{H}]$ uncertainty from ASPCAP analysis of combined spectrum \\
\hline$\$ 69$ & TI_H_ERR & Float & $\operatorname{dex}[\mathrm{Ti} / \mathrm{H}]$ uncertainty from ASPCAP analysis of combined spectrum \\
\hline$\$ 70$ & V_H_ERR & Float & dex $[\mathrm{V} / \mathrm{H}]$ uncertainty from ASPCAP analysis of combined spectrum \\
\hline$\$ 71$ & AL_H_FLAG & Integer & {$[\mathrm{Al} / \mathrm{H}]$ Flag } \\
\hline$\$ 72$ & CA_H_FLAG & Integer & {$[\mathrm{Ca} / \mathrm{H}]$ Flag } \\
\hline$\$ 73$ & C_H_FLAG & Integer & {$[\mathrm{C} / \mathrm{H}]$ Flag } \\
\hline$\$ 74$ & FE_H_FLAG & Integer & {$[\mathrm{Fe} / \mathrm{H}]$ Flag } \\
\hline$\$ 75$ & K_H_FLAG & Integer & {$[\mathrm{K} / \mathrm{H}]$ Flag } \\
\hline$\$ 76$ & MG_H_FLAG & Integer & {$[\mathrm{Mg} / \mathrm{H}]$ Flag } \\
\hline$\$ 77$ & MN_H_FLAG & Integer & {$[\mathrm{Mn} / \mathrm{H}]$ Flag } \\
\hline$\$ 78$ & NA_H_FLAG & Integer & {$[\mathrm{Na} / \mathrm{H}]$ Flag } \\
\hline$\$ 79$ & NI_H_FLAG & Integer & {$[\mathrm{Ni} / \mathrm{H}]$ Flag } \\
\hline$\$ 80$ & N_H_FLAG & Integer & {$[\mathrm{N} / \mathrm{H}]$ Flag } \\
\hline$\$ 81$ & O_H_FLAG & Integer & {$[\mathrm{O} / \mathrm{H}]$ Flag } \\
\hline$\$ 82$ & SI_H_FLAG & Integer & {$[\mathrm{Si} / \mathrm{H}]$ Flag } \\
\hline$\$ 83$ & S_H_FLAG & Integer & {$[\mathrm{S} / \mathrm{H}]$ Flag } \\
\hline$\$ 84$ & TI_H_FLAG & Integer & {$[\mathrm{Ti} / \mathrm{H}]$ Flag } \\
\hline$\$ 85$ & V_H_FLAG & Integer & {$[\mathrm{V} / \mathrm{H}]$ Flag } \\
\hline$\$ 86$ & A $\bar{K} \_\bar{T} A R G$ & Float & mag $K_{\mathrm{s}}$-band extinction from APOGEE Targeting (Zasowski et al. 2013) \\
\hline$\$ 87$ & AK_TARG_METHOD & String & Extinction method used for APOGEE Targeting (Zasowski et al. 2013) \\
\hline$\$ 88$ & SFD_EBV & Float & mag SFD $E(B-V)$ extinction (Schlegel et al. 1998) \\
\hline$\$ 89$ & RC_dist_kpc & Double & kpc APOGEE Red Clump distance (Bovy et al. 2014) \\
\hline$\$ 90$ & VISITS & String & List of APOGEE visits going into combined spectrum \\
\hline$\$ 91$ & VISIT_PK & int[] & Index of MJDs of APOGEE visits used in combined spectrum \\
\hline$\$ 92$ & mag_b & Float & mag OBSCAT Filter $B$ magnitude \\
\hline$\$ 93$ & mag_b_err & Float & mag OBSCAT Filter $B$ magnitude error \\
\hline$\$ 94$ & mag_v & Float & mag OBSCAT Filter $V$ magnitude \\
\hline$\$ 95$ & mag_v_err & Float & mag OBSCAT Filter $V$ magnitude error \\
\hline$\$ 96$ & mag_r & Float & mag OBSCAT Filter $R$ magnitude \\
\hline$\$ 97$ & mag_r_err & Float & mag OBSCAT Filter $R$ magnitude error \\
\hline$\$ 98$ & mag_i & Float & mag OBSCAT Filter $I$ magnitude \\
\hline$\$ 99$ & mag_i_err & Float & mag OBSCAT Filter $I$ magnitude error \\
\hline$\$ 10$ & O AllWISE & String & AllWISE ID \\
\hline$\$ 101$ & $1 \mathrm{Jmag}$ & Float & mag 2MASS $J$ magnitude \\
\hline$\$ 102$ & 2 e_Jmag & Float & mag Mean error on $J$ magnitude \\
\hline$\$ 103$ & $3 \mathrm{Hmag}$ & Float & mag 2MASS $H$ magnitude \\
\hline$\$ 10$ & 4 e_Hmag & Float & mag Mean error on $H$ magnitude \\
\hline$\$ 10$ & $5 \mathrm{Kmag}$ & Float & mag 2 MASS $K_{\mathrm{s}}$ magnitude \\
\hline$\$ 100$ & 6e_Kmag & Float & mag Mean error on $K_{\mathrm{s}}$ magnitude \\
\hline$\$ 10^{\prime}$ & $7 \mathrm{~W} 1 \mathrm{mag}$ & Float & mag WISE $W 1$ magnitude \\
\hline$\$ 10 \&$ & 8 e_W1mag & Float & mag Mean error on $W 1$ magnitude \\
\hline$\$ 10$ & $9 \mathrm{~W} 2 \mathrm{mag}$ & Float & mag WISE $W 2$ magnitude \\
\hline$\$ 110$ & e_w2mag & Float & mag Mean error on $W 2$ magnitude \\
\hline$\$ 11$ & $1 \mathrm{~W} 3 \mathrm{mag}$ & Float & mag WISE $W 3$ magnitude \\
\hline$\$ 112$ & $2 \mathrm{e} \_$W3mag & Float & mag Mean error on $W 3$ magnitude \\
\hline
\end{tabular}


Table B.1. continued.

\begin{tabular}{|c|c|c|c|}
\hline No. Column & Data type Shape & Unit & Description \\
\hline$\$ 113 \mathrm{~W} 4 \mathrm{mag}$ & Float & mag & WISE $W 4$ magnitude \\
\hline \$114 e_W4mag & Float & mag & Mean error on $W 4$ magnitude \\
\hline$\$ 115 \mathrm{magB}$ & Float & mag & $B$ magnitude from APASS \\
\hline$\$ 116$ sigmagB & Float & mag & Error in $B$ magnitude from APASS \\
\hline$\$ 117$ magV & Float & mag & $V$ magnitude from APASS \\
\hline$\$ 118$ sigmagV & Float & mag & Error in $V$ magnitude from APASS \\
\hline$\$ 119$ magg & Float & mag & $g$ magnitude from APASS \\
\hline$\$ 120$ sigmagg & Float & mag & Error in $g$ magnitude from APASS \\
\hline$\$ 121$ magr & Float & mag & $r$ magnitude from APASS \\
\hline$\$ 122$ sigmagr & Float & mag & Error in $r$ magnitude from APASS \\
\hline$\$ 123$ magi & Float & mag & $i$ magnitude from APASS \\
\hline$\$ 124$ sigmagi & Float & mag & Error in $i$ magnitude from APASS \\
\hline \$125 RA_EXODAT & Double & deg & Rigth Ascension - J2000 decimal degrees \\
\hline \$126 DEC_EXODAT & Double & deg & Declination - J2000 decimal degrees \\
\hline$\$ 127$ ccd_id & String & & CoRoT CCD Id. either E1 or E2 \\
\hline \$128win_id & Integer & & CoRoT Window Identifier on the CCD \\
\hline$\$ 129 x$ & Double & & $\mathrm{X}$ Target coordinate on the CoRoT CCD \\
\hline$\$ 130 y$ & Double & & Y Target coordinate on the CoRoT CCD \\
\hline \$131 spectral_type & String & & EXODAT Spectral type of the star (from SED) \\
\hline \$132 luminosity_class & String & & EXODAT Luminosity class - (I II III IV V) (from SED) \\
\hline$\$ 133$ color_temperature & Integer & $\mathrm{K}$ & EXODAT Color temperature estimation \\
\hline \$134 ppmxl_id & String & & Record identifier in the catalog PPMXL \\
\hline$\$ 135$ usnob1_id & String & & Record identifier in the catalog USNO-B1 \\
\hline \$136 twomass_id & String & & Record identifier in the catalog TWOMASS \\
\hline \$137 usnoa2_id & String & & Record identifier in the catalog USNO-A2 \\
\hline$\$ 138$ cmc14_id & String & & Record identifier in the catalog CMC-14 \\
\hline \$139 tycho2_id & Integer & & Record identifier in the catalog TYCHO-2 \\
\hline$\$ 140$ ucac2_id & Integer & & Record identifier in the catalog UCAC- 2 \\
\hline \$141 UCAC̄ 4 & String & & UCAC4 recommended identifier (ZZZ-NNNNNN) \\
\hline$\$ 142$ pmRA & Double & $\mathrm{mas} / \mathrm{yr}$ & UCAC4 Proper motion in RA $\cdot \cos (\mathrm{Dec})$ \\
\hline$\$ 143$ e_pmRA & Float & $\mathrm{mas} / \mathrm{yr}$ & Mean error on pmRA \\
\hline$\$ 144 \mathrm{pmDE}$ & Double & $\mathrm{mas} / \mathrm{yr}$ & UCAC4 Proper motion in Dec \\
\hline$\$ 145$ e_pmDE & Float & $\mathrm{mas} / \mathrm{y} 1$ & Mean error on $\mathrm{pmDE}$ \\
\hline \$146 UCAC4_combinedflag & Boolean & & UCAC4 combined flag (as in Anders et al. 2014) \\
\hline$\$ 147$ mass_scale & Float & $M_{\odot}$ & Stellar mass from seismic scaling relations \\
\hline$\$ 148$ logg_scale & Float & {$[\mathrm{dex}]$} & $\log g$ from seismic scaling relations \\
\hline$\$ 149$ radius_scale & Float & $R_{\odot}$ & Stellar radius from seismic scaling relations \\
\hline$\$ 150$ age_mode & Float & Gyr & PARAM Age (mode of the posterior PDF; Rodrigues et al. 2014) \\
\hline \$151 age_mode_68L & Float & Gyr & PARAM Age Lower $68 \%$ Confidence Limit \\
\hline$\$ 152$ age_mode_68U & Float & Gyr & PARAM Age Upper $68 \%$ Confidence Limit \\
\hline$\$ 153$ age_mode_95L & Float & Gyr & PARAM Age Lower $95 \%$ Confidence Limit \\
\hline \$154 age_mode_95U & Float & Gyr & PARAM Age Upper 95\% Confidence Limit \\
\hline \$155 sigage_isochrones & Double & Gyr & Systematic age uncertainty estimated from different isochrones \\
\hline \$156 sigage_eta & Double & Gyr & Systematic age uncertainty related to mass loss \\
\hline$\$ 157$ sigage_tot & Double & Gyr & $\begin{array}{l}\text { Total age uncertainty } \\
\text { (quadratic sum of statistical and systematic age uncertainties) }\end{array}$ \\
\hline \$158 mass_mode & Float & $M_{\odot}$ & PARAM Mass (mode of the posterior PDF; Rodrigues et al. 2014) \\
\hline \$159 mass_mode_68L & Float & $M_{\odot}$ & PARAM Mass Lower $68 \%$ Confidence Limit \\
\hline \$160 mass_mode_68U & Float & $M_{\odot}$ & PARAM Mass Upper $68 \%$ Confidence Limit \\
\hline \$161 mass_mode_95L & Float & $M_{\odot}$ & PARAM Mass Lower 95\% Confidence Limit \\
\hline \$162 mass_mode_95U & Float & $M_{\odot}$ & PARAM Mass Upper 95\% Confidence Limit \\
\hline$\$ 163$ radius_mode & Float & $R_{\odot}$ & PARAM Radius (mode of the posterior PDF; Rodrigues et al. 2014) \\
\hline$\$ 164$ radius_mode_68L & Float & $R_{\odot}$ & PARAM Radius Lower $68 \%$ Confidence Limit \\
\hline$\$ 165$ radius_mode_68U & Float & $R_{\odot}$ & PARAM Radius Upper $68 \%$ Confidence Limit \\
\hline$\$ 166$ radius_mode_95L & Float & $R_{\odot}$ & PARAM Radius Lower 95\% Confidence Limit \\
\hline$\$ 167$ radius_mode_95U & Float & $R_{\odot}$ & PARAM Radius Upper 95\% Confidence Limit \\
\hline$\$ 168$ logg_mode & Float & {$[\mathrm{dex}]$} & PARAM $\log g$ (mode of the posterior PDF; Rodrigues et al. 2014) \\
\hline \$169 logg_mode_68L & Float & {$[\mathrm{dex}]$} & PARAM $\log g$ Lower $68 \%$ Confidence Limit \\
\hline$\$ 170$ logg_mode_68U & Float & {$[\mathrm{dex}]$} & PARAM $\log g$ Upper $68 \%$ Confidence Limit \\
\hline \$171 logg_mode_95L & Float & {$[\mathrm{dex}]$} & PARAM $\log g$ Lower $95 \%$ Confidence Limit \\
\hline
\end{tabular}


Table B.1. continued.

\begin{tabular}{|c|c|c|c|}
\hline No. Column & Data type Shape & Unit & Description \\
\hline \$172logg_mode_95U & Float & {$[\mathrm{dex}]$} & PARAM $\log g$ Upper 95\% Confidence Limit \\
\hline$\$ 173$ dist_mode & Float & $\mathrm{pc}$ & PARAM Distance (mode of the posterior PDF; Rodrigues et al. 2014) \\
\hline \$174 dist_mode_68L & Float & $\mathrm{pc}$ & PARAM Distance Lower $68 \%$ Confidence Limit \\
\hline$\$ 175$ dist_mode_68U & Float & $\mathrm{pc}$ & PARAM Distance Upper $68 \%$ Confidence Limit \\
\hline \$176 dist_mode_95L & Float & $\mathrm{pc}$ & PARAM Distance Lower 95\% Confidence Limit \\
\hline$\$ 177$ dist_mode_95U & Float & $\mathrm{pc}$ & PARAM Distance Upper 95\% Confidence Limit \\
\hline$\$ 178$ Av_mode & Float & mag & PARAM $V$-band extinction (mode of the posterior PDF; Rodrigues et al. 2014) \\
\hline \$179 Av_mode_68L & Float & mag & PARAM $V$-band extinction Lower $68 \%$ Confidence Limit \\
\hline \$180 Av_mode_68U & Float & mag & PARAM $V$-band extinction Upper $68 \%$ Confidence Limit \\
\hline \$181 Av_mode_95L & Float & mag & PARAM $V$-band extinction Lower $95 \%$ Confidence Limit \\
\hline \$182 Av_mode_95U & Float & mag & PARAM $V$-band extinction Upper 95\% Confidence Limit \\
\hline$\$ 183$ nfil & Short & & Number of photometric filters used by PARAM \\
\hline$\$ 184$ fils & String & & Which photometric filters have been used by PARAM \\
\hline$\$ 185 \mathrm{Xg}$ & Float & $\mathrm{kpc}$ & $X$ coordinate in Galactocentric Cartesian coordinates \\
\hline \$186 Xg_sig & Float & $\mathrm{kpc}$ & $X$ coordinate uncertainty \\
\hline$\$ 187 \mathrm{Yg}$ & Float & $\mathrm{kpc}$ & $Y$ coordinate in Galactocentric Cartesian coordinates \\
\hline \$188 Yg_sig & Float & $\mathrm{kpc}$ & $Y$ coordinate uncertainty \\
\hline$\$ 189 \mathrm{Zg}$ & Float & $\mathrm{kpc}$ & $Z$ coordinate in Galactocentric Cartesian coordinates \\
\hline \$190Zg_sig & Float & $\mathrm{kpc}$ & $Z$ coordinate uncertainty \\
\hline$\$ 191 \mathrm{Rg}$ & Float & $\mathrm{kpc}$ & $R$ coordinate in Galactocentric Cylindrical coordinates \\
\hline \$192 Rg_sig & Float & $\mathrm{kpc}$ & $R$ coordinate uncertainty \\
\hline$\$ 193 \mathrm{vT}$ & Float & $\mathrm{km} \mathrm{s}^{-1}$ & Transverse velocity \\
\hline \$194 vT_sig & Float & $\mathrm{km} \mathrm{s}^{-1}$ & Transverse velocity uncertainty \\
\hline$\$ 195 \mathrm{vXg}$ & Float & $\mathrm{km} \mathrm{s}^{-1}$ & $X$-axis space velocity \\
\hline \$196 vXg_sig & Float & $\mathrm{km} \mathrm{s}^{-1}$ & $X$-axis space velocity uncertainty \\
\hline$\$ 197$ vYg & Float & $\mathrm{km} \mathrm{s}^{-1}$ & $Y$-axis space velocity \\
\hline \$198vYg_sig & Float & $\mathrm{km} \mathrm{s}^{-1}$ & $Y$-axis space velocity uncertainty \\
\hline$\$ 199 \mathrm{vZg}$ & Float & $\mathrm{km} \mathrm{s}^{-1}$ & $Z$-axis space velocity \\
\hline \$200 vZg_sig & Float & $\mathrm{km} \mathrm{s}^{-1}$ & $Z$-axis space velocity uncertainty \\
\hline$\$ 201 \mathrm{vRg}$ & Float & $\mathrm{km} \mathrm{s}^{-1}$ & Galactocentric radial velocity \\
\hline \$202 vRg_sig & Float & $\mathrm{km} \mathrm{s}^{-1}$ & Galactocentric radial velocity uncertainty \\
\hline$\$ 203$ vPhig & Float & $\mathrm{km} \mathrm{s}^{-1}$ & Azimuthal velocity \\
\hline \$204 vPhig_sig & Float & $\mathrm{km} \mathrm{s}^{-1}$ & Azimuthal velocity uncertainty \\
\hline \$205 Rguide & Float & $\mathrm{kpc}$ & Guiding-centre radius \\
\hline \$206 Rguide_sig & Float & $\mathrm{kpc}$ & Uncertainty on guiding-centre radius \\
\hline
\end{tabular}

